\title{
Discovery of extremely halophilic, methyl-reducing euryarchaea provides insights into the evolutionary origin of methanogenesis
}

Dimitry Y. Sorokin, Kira S. Makarova, Ben Abbas, Manuel Ferrer, Peter N. Golyshin, Erwin A. Galinski, Sergio Ciordia, María Carmen Mena, Alexander Y. Merkel, Yuri I. Wolf, Mark C.M. van Loosdrecht, Eugene V. Koonin

\section{Content:}

\section{Supplementary Tables 1 and 2,}

\section{Supplementary Figures 1-10,}

\section{Supplementary Tables (provided as separate Excel files):}

Table 3. Comparative genomic analysis based on arCOG assignments. The Table provides complete list of arCOGs present in AMET1 and HMET1 and two groups of interest: halobacteriales and methanogenic archaea. arCOGs shown on the Figure 6 are mapped. Ribosomal proteins used for phylogeny reconstruction are mapped. Archaeal core genes are mapped. Gene sets used for multidimensional scaling analysis are mapped. Results of proteomic analysis are shown. Genes known to be involved in central metabolic pathways are shown together with indicated of the step of the respective pathway.

Table 4. Reconstruction of gene gain and loss.

Table 5. Isoelectric point calculation data.

Table 6. Proteomics analysis for AMET1. Identified proteins are sorted by emPAI index.

Table 7. Proteomics analysis for HMET1. Identified proteins are sorted by emPAI index.

\section{Supplementary Data 1-4 includes original trees in Newick format (provided below)}


Supplementary Data 3. Phylogenetic tree of McrA subunit (FastTree method) in Newick. The maximum-likelihood phylogenetic tree of the McrA proteins was built using the FastTree program as described in the Materials and Methods section. Sequence set and underlying multiple alignment, which can be provided by a request, are the same as on the Supplementary Fig. 9. The tree (below) is provided in Newick format, could be open in MEGA or in any other tree visualization software. To open the file copy the text below the dotted line to a text file with "tre" extension. MEGA (or other tree visualization software) should open the file automatically.

Supplementary Data 4. Phylogenetic tree of McrA subunit (RAxML method) in Newick. The maximum-likelihood phylogenetic tree of the McrA proteins was built using the RAxML program as described in the Materials and Methods section. Sequence set and underlying multiple alignment, which can be provided by a request, are the same as on the Supplementary Fig. 9. The tree (below) is provided in Newick format, could be open in MEGA or in any other tree visualization software. To open the file copy the text below the dotted line to a text file with "tre" extension. MEGA (or other tree visualization software) should open the file automatically. 
Supplementary Table 1. Key chemical characteristics of investigated hypersaline lakes

\begin{tabular}{|c|c|c|c|c|c|c|c|c|}
\hline \multirow[t]{2}{*}{ Lake } & \multicolumn{2}{|l|}{ Location } & \multirow{2}{*}{$\begin{array}{l}\text { Sampling } \\
\text { year }\end{array}$} & \multicolumn{3}{|l|}{ Brines } & \multicolumn{2}{|l|}{ Sediments } \\
\hline & Area & Coordinates & & $\begin{array}{l}\text { Total } \\
\text { salts }(g / l)\end{array}$ & $\mathrm{pH}$ & $\begin{array}{l}\text { Soluble carbonate } \\
\text { alkalinity (M) }\end{array}$ & $\begin{array}{l}\mathrm{HS}^{-}+\mathrm{FeS} \\
\mathrm{mM}\end{array}$ & $\begin{array}{l}\mathrm{CH}_{4} \\
\mu \mathrm{M}\end{array}$ \\
\hline \multicolumn{9}{|c|}{ Hypersaline salt lakes } \\
\hline Cock Salt Lake & \multirow{3}{*}{$\begin{array}{l}\begin{array}{l}\text { Kulunda Steppe } \\
\text { (Altai, Russia) }\end{array} \\
\end{array}$} & $\mathrm{N} 52^{\circ} 16^{\prime} / \mathrm{E} 79^{\circ} 52^{\prime}$ & \multirow[t]{4}{*}{2015} & $280-320$ & $7.5-7.7$ & - & & \\
\hline Lake Lomovoe & & $\mathrm{N} 51^{\circ} 42^{\prime} / \mathrm{E} 79^{\circ} 42^{\prime}$ & & $300-340$ & $7.9-8.1$ & - & & \\
\hline Hummocky Lake & & $\mathrm{N} 51^{\circ} 42^{\prime} / \mathrm{E} 79^{\circ} 56^{\prime}$ & & $300-350$ & $8.0-8.1$ & - & & \\
\hline Lake Elton & \multirow[t]{2}{*}{ South Russia } & $\mathrm{N} 49^{\circ} 10^{\prime} / \mathrm{E} 46^{\circ} 39^{\prime}$ & & 320 & 6.7 & - & nd & nd \\
\hline Lake Baskunchak & & $\mathrm{N} 48^{\circ} 14^{\prime} / \mathrm{E} 46^{\circ} 35^{\prime}$ & 2013 & 360 & 6.2 & - & nd & nd \\
\hline Eupatorian solar saltern & Crimea (Russia) & $\mathrm{N} 45^{\circ} 11^{\prime} / \mathrm{E} 33^{\circ} 19^{\prime}$ & 2015 & 220 & 7.2 & - & nd & nd \\
\hline \multicolumn{9}{|c|}{ Hypersaline alkaline and soda lakes } \\
\hline Tanatar-1 & \multirow{5}{*}{$\begin{array}{l}\text { Kulunda Steppe } \\
\text { (Altai, Russia) }\end{array}$} & $\mathrm{N} 51^{\circ} 39^{\prime} / \mathrm{E}^{\prime} 79^{\circ} 48^{\prime}$ & \multirow{5}{*}{$\begin{array}{l}2013- \\
2015\end{array}$} & 380 & 10.25 & 3.4 & & \\
\hline Bitter-1 & & $\mathrm{N} 51^{\circ} 40^{\prime} / \mathrm{E} 79^{\circ} 54^{\prime}$ & & $120-220$ & 9.6-10.3 & $1.2-3.8$ & & \\
\hline Trona crystallizer & & $\mathrm{N} 51^{\circ} 39^{\prime} / \mathrm{E} 79^{\circ} 46^{\prime}$ & & $350-400$ & $9.6-10.2$ & $3.8-5.2$ & & \\
\hline Cock Soda lake & & $\mathrm{N} 52^{\circ} 06^{\prime} / \mathrm{E} 79^{\circ} 09^{\prime}$ & & $50-190$ & $9.6-10.1$ & $0.5-0.65$ & $0.6-15$ & $0.7-20$ \\
\hline Stamp Lake & & $\mathrm{N} 51^{\circ} 41^{\prime} / \mathrm{E} 79^{\circ} 46^{\prime}$ & & $240-320$ & 9.1 & 0.15 & & \\
\hline $\begin{array}{l}\text { Hamra, Fazda, Beidah, } \\
\text { Gaar, Umm-Risha, } \\
\text { Zugm, Ruzita, Khadra }\end{array}$ & $\begin{array}{l}\text { Wadi al Natrun } \\
\text { (Libyan Desert, } \\
\text { Egypt) }\end{array}$ & $\mathrm{N} 30^{\circ} 24^{\prime} / \mathrm{E} 30^{\circ} 18^{\prime}$ & 2000 & $200-360$ & $9.1-9.9$ & $0.1-0.9$ & nd & nd \\
\hline Searles Lake & California (USA) & $\mathrm{N} 35^{\circ} 44^{\prime} / \mathrm{W} 117^{\circ} 20^{\prime}$ & 2005 & 350 & 9.8 & 0.2 & nd & nd \\
\hline
\end{tabular}


Supplementary Table 2.

Extremely halophilic and moderately thermophilic mixotrophic methanogens isolated from hypersaline lakes at $4 \mathrm{M}$ total Na $^{+}$

\begin{tabular}{|c|c|c|c|c|c|c|c|c|c|c|}
\hline \multirow[b]{2}{*}{ Strain } & \multirow[b]{2}{*}{ Lake } & \multirow[b]{2}{*}{ Area } & \multicolumn{3}{|c|}{ Enrichment conditions } & \multicolumn{2}{|c|}{ Growth parameters } & \multicolumn{2}{|c|}{$\%$ similarity to AMET1 } & \multirow{2}{*}{$\begin{array}{c}\text { Culture collection } \\
\text { deposition }\end{array}$} \\
\hline & & & Substrate & $\mathrm{pH}$ & $\mathrm{T},{ }^{\circ} \mathrm{C}$ & $\begin{array}{l}\mathrm{T}_{\max },{ }^{\circ} \mathrm{C} \\
\text { (activity) }\end{array}$ & $\begin{array}{c}\text { Dependence } \\
\text { on }\end{array}$ & $\begin{array}{l}\text { 16S rRNA } \\
\text { gene }\end{array}$ & mcrA & \\
\hline AMET1 & Mix 5 soda lakes & $\begin{array}{c}\text { Kulunda Steppe } \\
\text { (Altai, Russia) } \\
\text { 2013-2015 }\end{array}$ & $\mathrm{MeOH}+$ formate & \multirow{8}{*}{9.6} & 48 & 55 & \multirow[t]{3}{*}{$\mathrm{FeS}$} & 100 & 100 & $\begin{array}{c}\text { DSM } 28684 \\
\text { NBRC } 110805 \\
\text { UNIQEM } 982 \\
\end{array}$ \\
\hline AMET3 & Tanatar-1 & & & & 48 & 62 & & 99.6 & 99 & UNIQEM 985 \\
\hline AMET4 & Picturesque Lake & & & & 48 & 50 & & 99.5 & 99 & UNIQEM 986 \\
\hline AMET5 & Mix 6 soda lakes & & TMA +formate & & 48 & 50 & \multirow[t]{8}{*}{ sediments } & 99.5 & 99 & UNIQEM 987 \\
\hline AMET6-2 & Tanatar-1 & & $\mathrm{MeOH}+$ formate & & 60 & 65 & & 98.4 & 98.2 & UNIQEM 988 \\
\hline AMET7 & Soda crystallizer & & & & 55 & 62 & & 99.5 & 100 & UNIQEM 989 \\
\hline AMET8 & Mix 6 soda lakes & & & & 30 & 55 & & 99.6 & 99 & \\
\hline AMET9 & Soda crystallizer & & & & 43 & 60 & & 100 & 99 & \\
\hline AMET10 & Stamp Lake & & & 9.2 & 54 & 62 & & 99.6 & 99 & \\
\hline AMET2 & $\begin{array}{c}\text { Mix from } 8 \\
\text { hypersaline } \\
\text { alkaline lakes }\end{array}$ & $\begin{array}{l}\text { Wadi al Natrun } \\
\text { (Egypt, 2000) }\end{array}$ & \multirow[t]{2}{*}{$\mathrm{MeOH}+$ formate } & \multirow[t]{2}{*}{9.6} & 60 & 62 & & 99.5 & 100 & UNIQEM 984 \\
\hline AMET-SI & Searles Lake & California & & & 48 & 50 & & 98.2 & & UNIQEM 1004 \\
\hline $\begin{array}{c}\text { HMET1 } \\
\text { (mixed culture) }\end{array}$ & $\begin{array}{c}\text { Mix from } 4 \\
\text { salt lakers }\end{array}$ & $\begin{array}{c}\text { Kulunda Steppe } \\
2014 \\
\end{array}$ & $\mathrm{TMA}+\mathrm{H}_{2}$ & \multirow{3}{*}{7.0} & 48 & 55 & \multirow{3}{*}{ sediments } & 90 & 76 & \\
\hline $\begin{array}{c}\text { HMET-EI } \\
\text { (mixed culture) }\end{array}$ & Lake Elton & $\begin{array}{c}\text { Southa Russia } \\
2015\end{array}$ & \multirow{2}{*}{$\mathrm{MeOH}+$ formate } & & 54 & 55 & & 90 & 78 & \\
\hline $\begin{array}{c}\text { HMET-Eu } \\
\text { (mixed culture) }\end{array}$ & Salt crystallizer & $\begin{array}{c}\text { Crimea (Russia) } \\
2015\end{array}$ & & & 55 & 55 & & 90 & 78 & \\
\hline
\end{tabular}



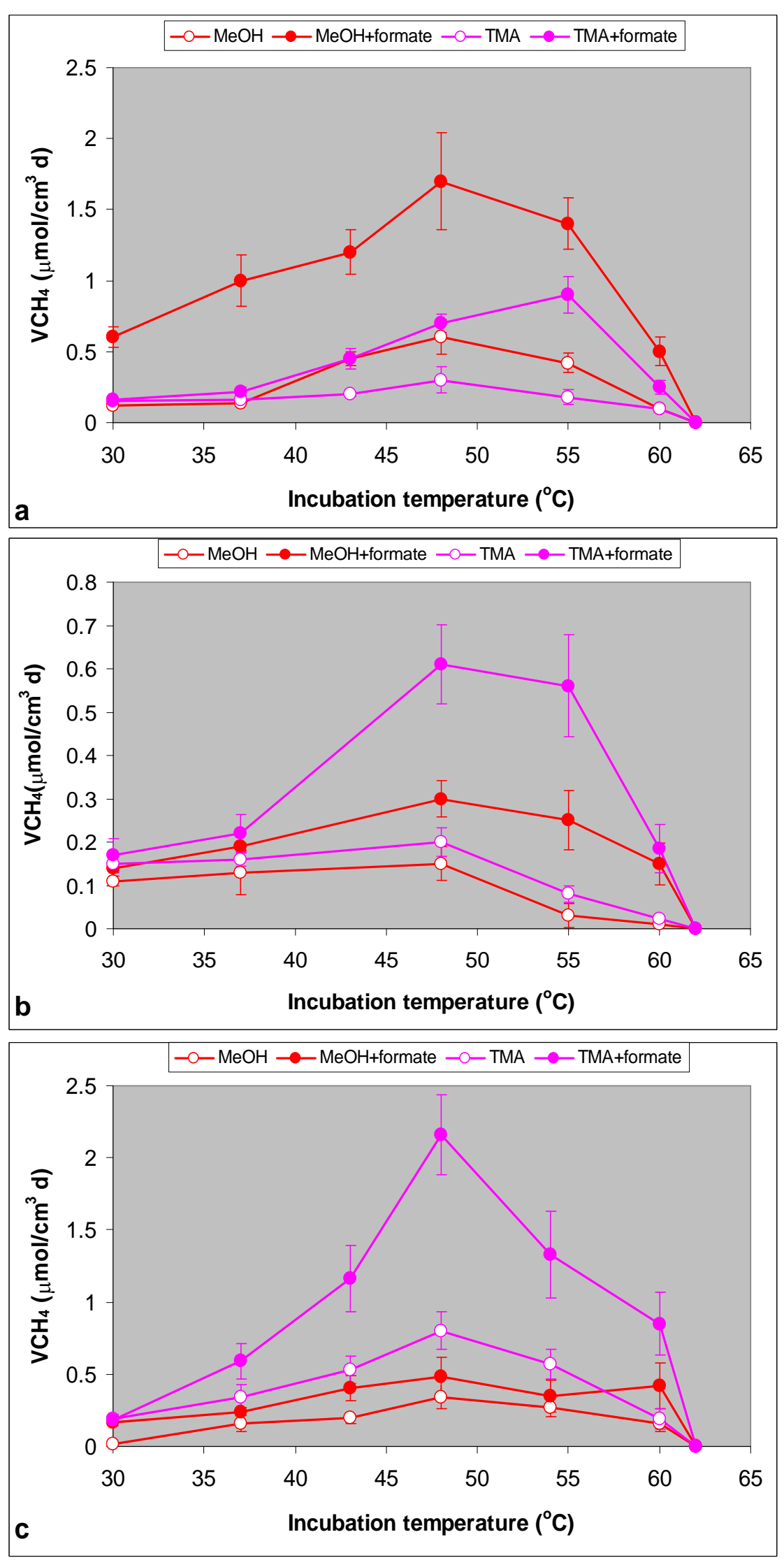

Supplementary Figure 1 Influence of temperature on potential methanogenic activity of anoxic sedimentbrine slurries from 3 different hypersaline systems: (a) - hypersaline soda lake Tanatar-1 (Kulunda Steppe, Altai, Russia); (b) - mixed sediments from 4 hypersaline salt lakes (Kulunda Steppe, Altai, Russia); (c) - sea salt evaporative concentrator (Eupatoria, Crimea, Russia). The error bars are SD from biological replicates $(n=2)$. $\mathrm{VCH} 4$ is the specific rate of methane formation. 


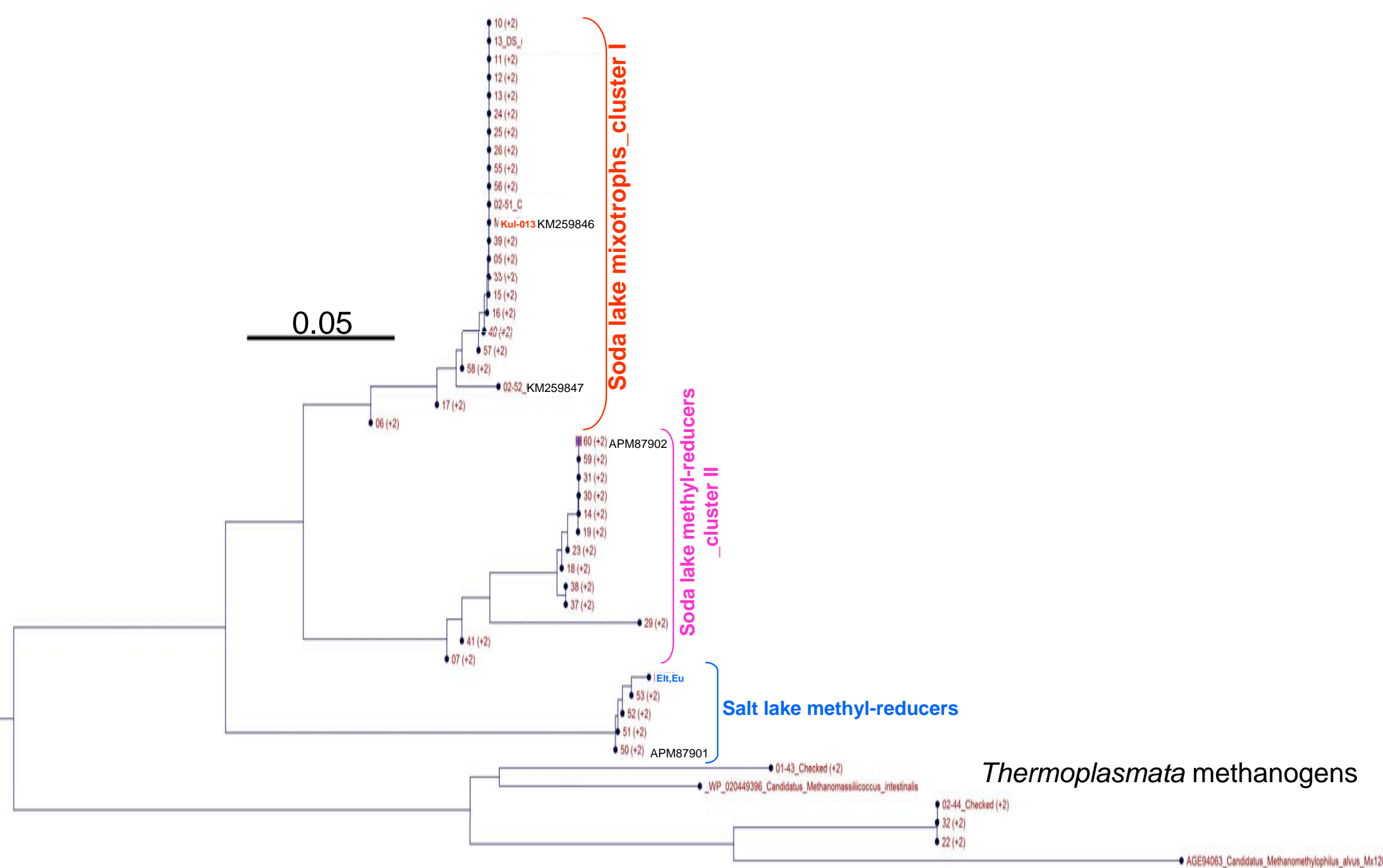

Supplementary Figure 2 McrA-DGGE phylogenetic results of sediment incubation experiments at high temperature. Kul-013 - a reference sequence found previously in Kulunda soda lakes in 2013; Elt-Eu - sequences from high temperature enrichments on Me+formate from the salt lake Elton (south Russia) and the sea solar saltern in Eupatoria (Crimea). 


\section{$\mathrm{CH}_{4}$}

AMET1: biofilm formation in hydrotroillite and its disturbance by $\mathrm{CH}_{4}$ bubbles
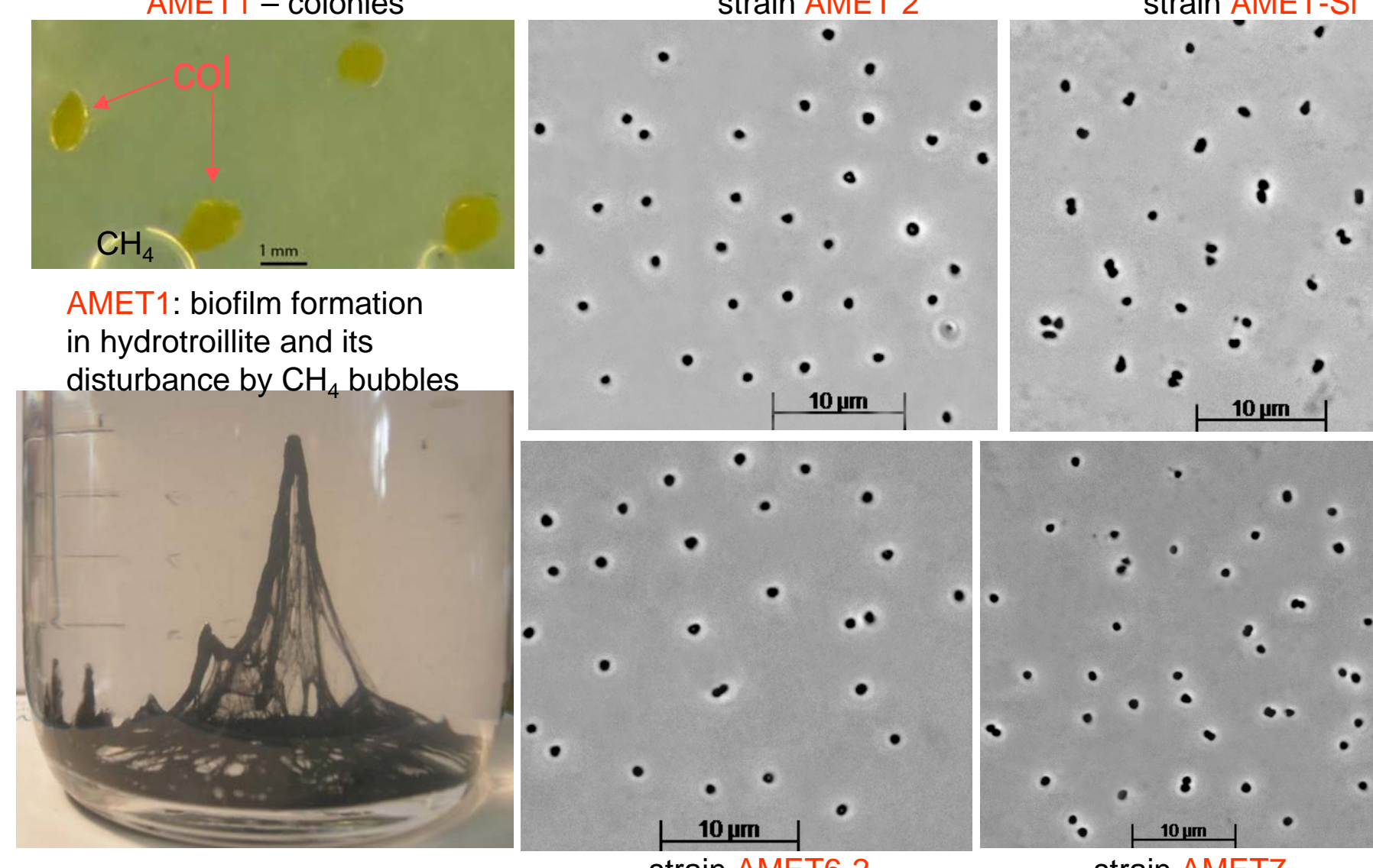

strain AMET7

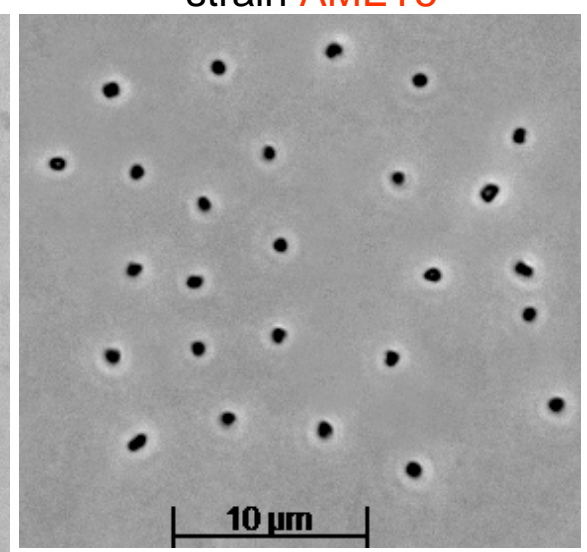

Supplementary Figure 3

Morphology of methyl-reducing methanogens from hypersaline lakes. The images are typical of 5 technical replicates of single cultures.

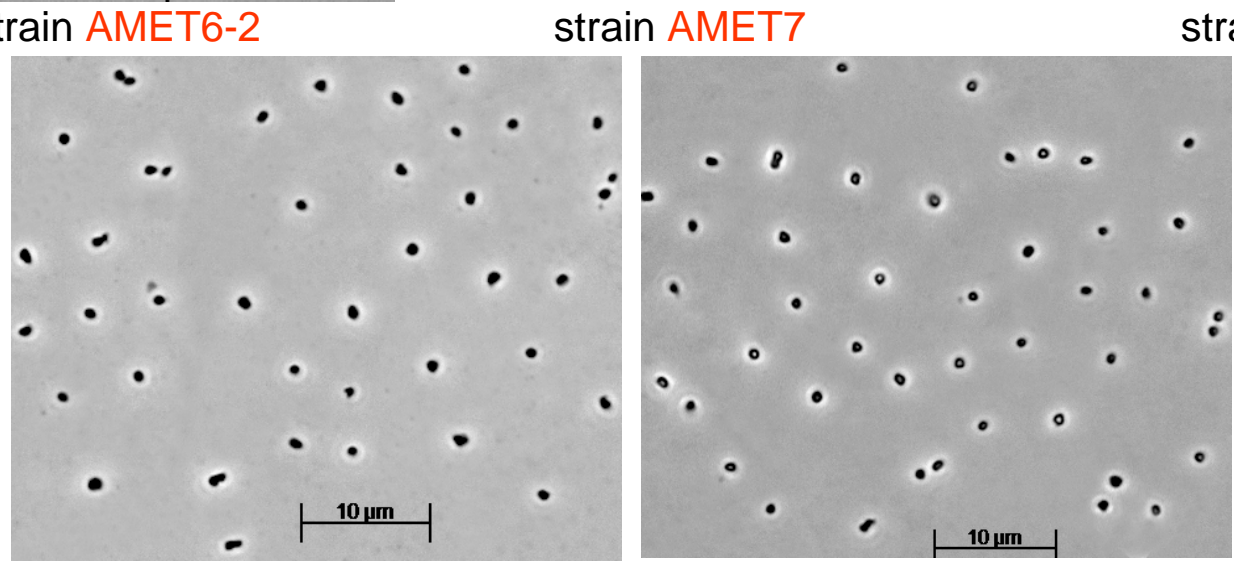




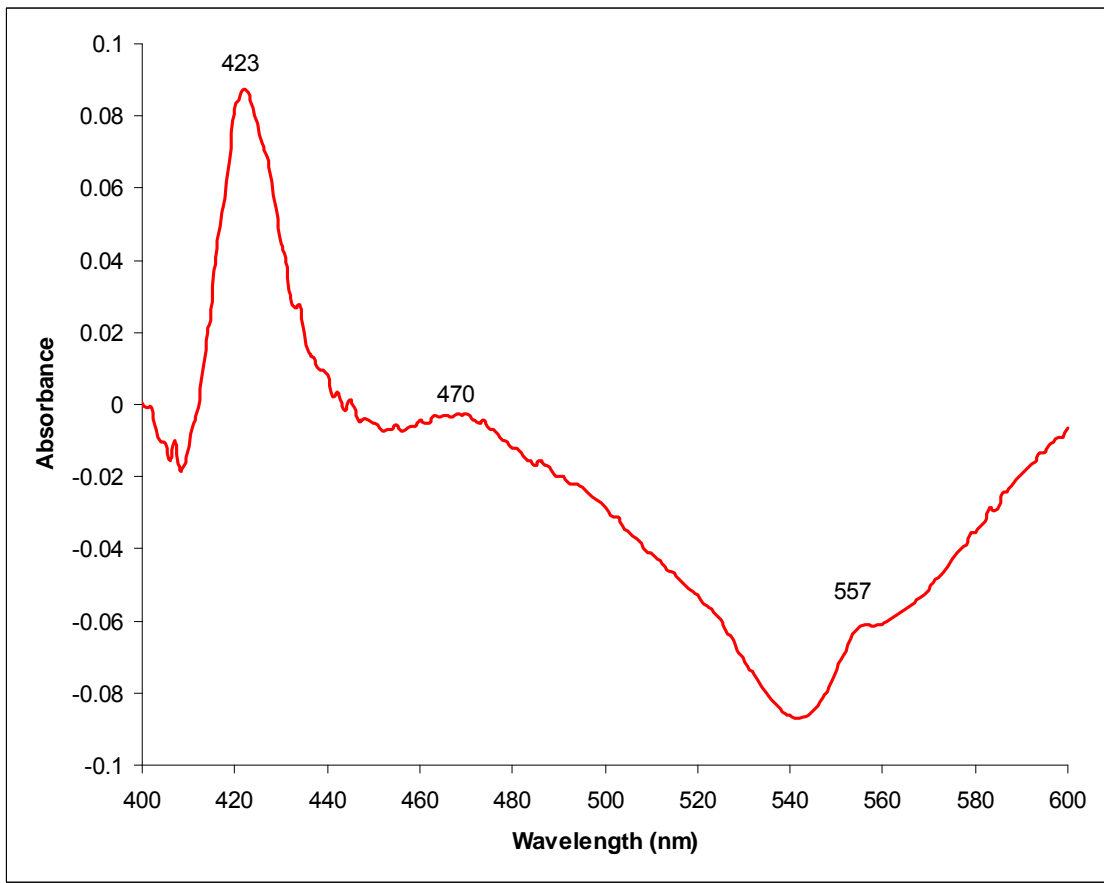

a.

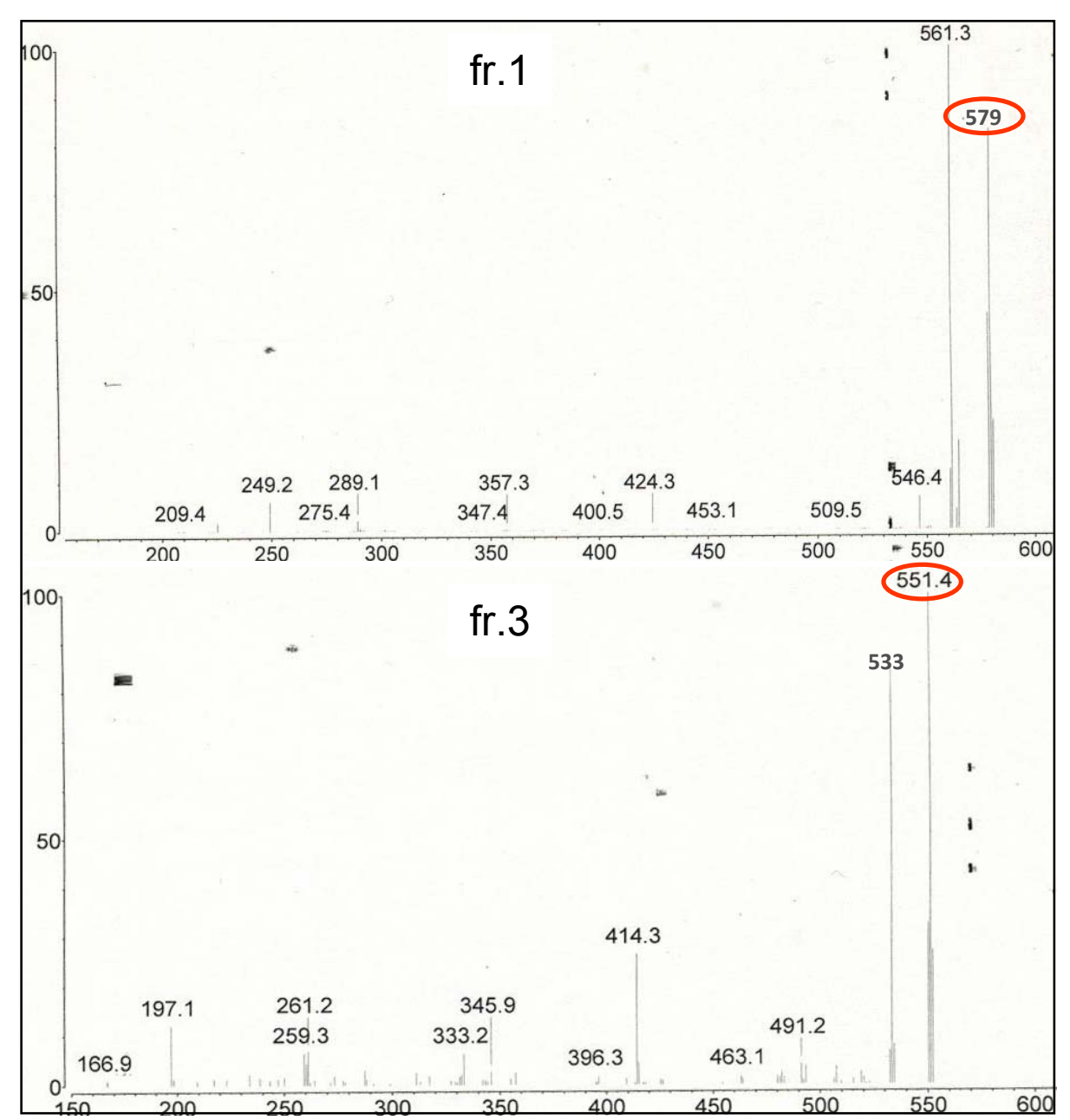

b.

Supplementary Figure 4 Elements of membrane-bound respiratory chain of strain AMET1. (a) Dithionite-reduced minus air-oxidized difference cytochrome spectrum of cell-free extract $(2 \mathrm{mg}$ protein $/ \mathrm{ml})$ prepared from cells of strain AMET1 grown with sterile sediments at $4 \mathrm{M}$ total $\mathrm{Na}+$, pH 9.8 , $480 \mathrm{C}$ and with $\mathrm{MeOH}+$ formate. The $\mathrm{y}$-peak at $423 \mathrm{~nm}$ and the a-peak at $557 \mathrm{~nm}$ belong to reduced cytochrome b. (b) Chemical ionization mass spectra of the quinone-like compounds extracted from cells of strain AMET1 and separated in 3 fraction. Fraction 2 contained too little material and was not analyzed. The results represent data from single sample analysis. 

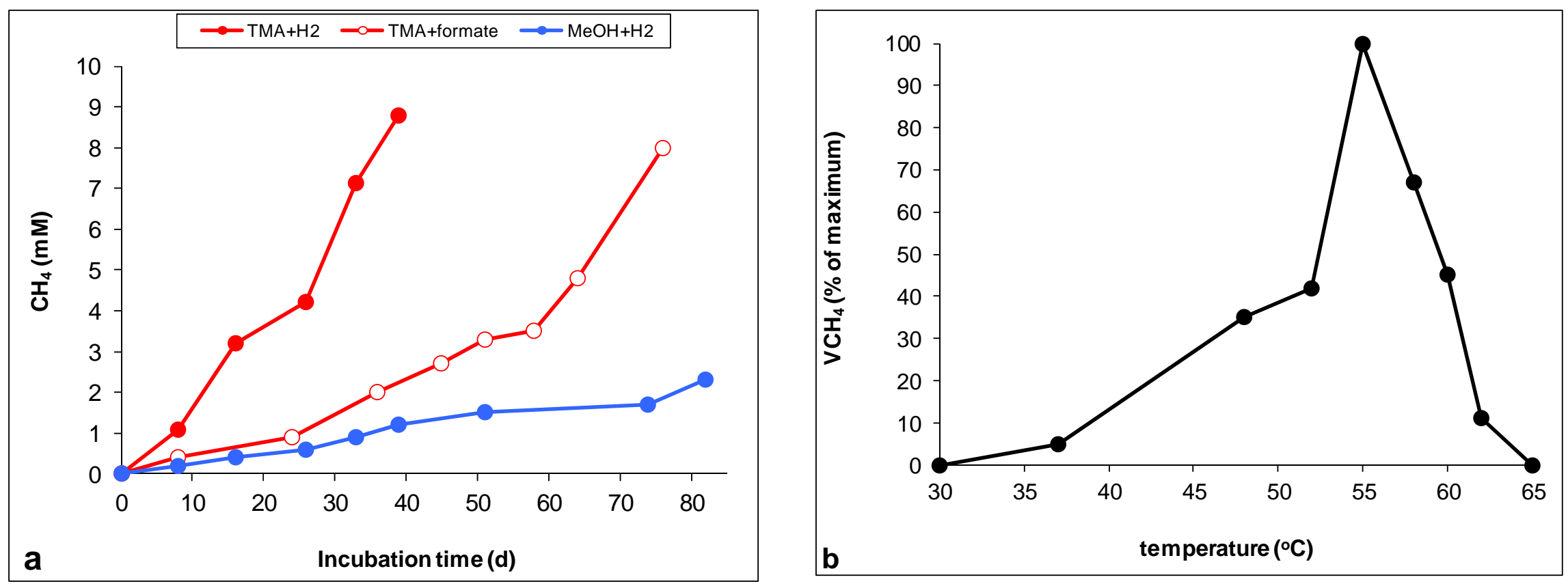

Supplementary Figure 5 Growth dynamics at $50^{\circ} \mathrm{C}$ (a) and temperature profile of methanogenic activity of washed cells (b) of HMET1 with TMA $+\mathrm{H} 2$ at $4 \mathrm{M} \mathrm{NaCl}$. No growth and no activity were observed with mono-substrates (i.e. TMA, MeOH, $\mathrm{H} 2$ or formate alone). Results of single experiment. 

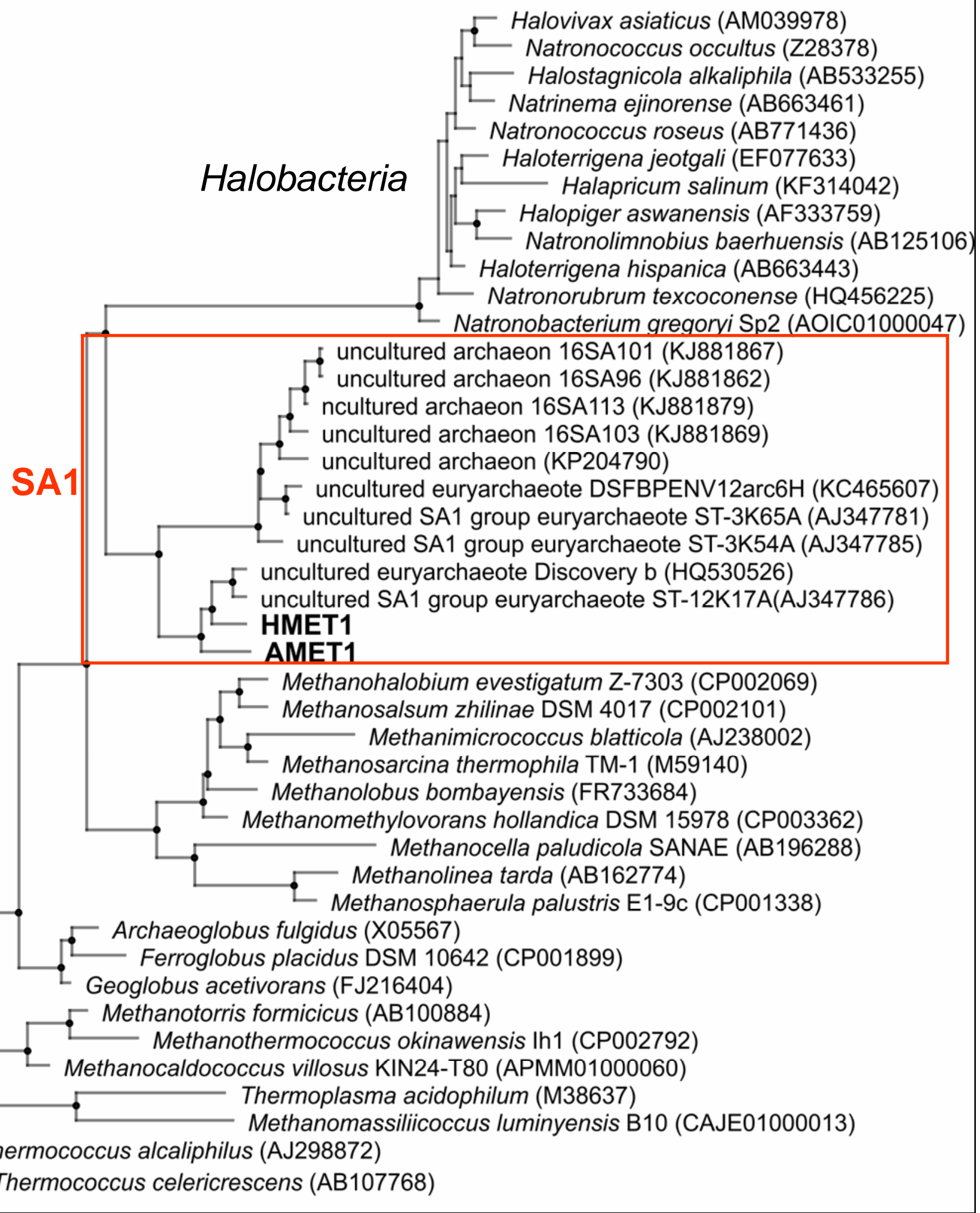

Supplementary Figure 6 16S-rRNA gene-based phylogenetic tree showing position of the uncultured SA1 group, including the methyl-reducing methanogens from hypersaline lakes within the Euryarchaeota. The tree was reconstructed using the ML algorithm (RAxML in ARBv5.2), 1214 positions used with a SSUref: archaea position variability filter. The nods indicate the bootstrap values (250 rounds) above $90 \%$. 


\section{Methyltrasferases AMET1}
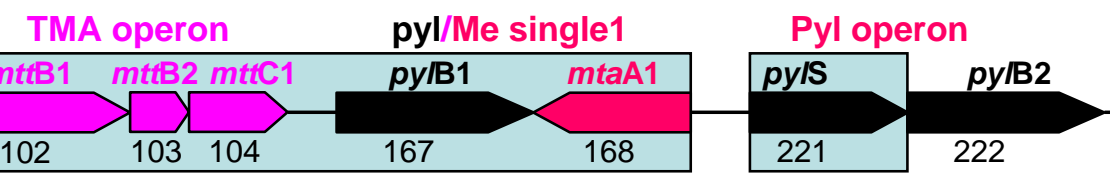

DMA operon1

DMA single1

MA/pyl operon

$\sum_{334}^{m t b C 1} \sum_{335}^{m t b A 1}$

mixed DMA-TMA-Me operon

\section{Methyltrasferases HMET1}

DMA-MA-pyl operon

TMA-DMA-pyl operon

DMA operon1

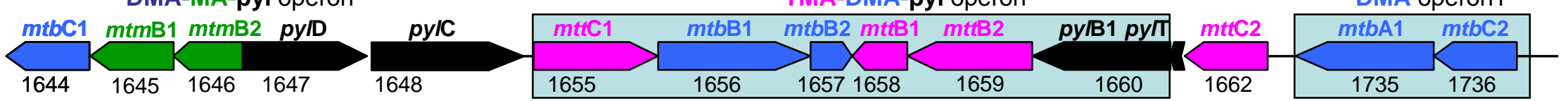

DMA operon2 DMA operon3

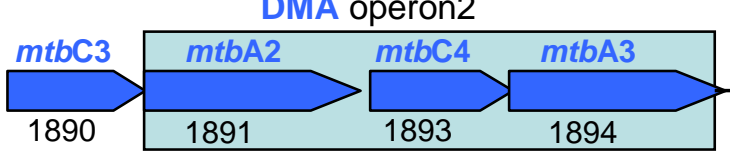

TMA operon1

$\overbrace{2215}^{m+m+C 3}$

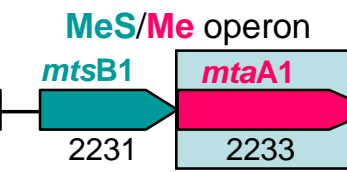

Me single1

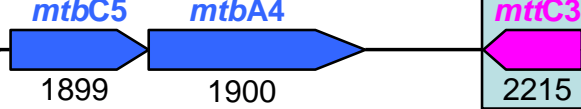

2216

2231

2233

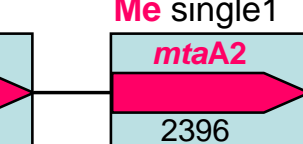

Me operon

TMA operon2

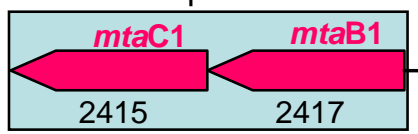

\begin{tabular}{|l}
$m t t B 4$ \\
\hline 2420
\end{tabular} mttB5

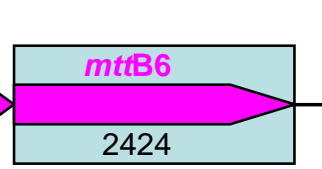

DMA operon4

\begin{tabular}{|l|l|}
\hline$m$ mtbA5 & $m$ mtbC6 \\
\hline & \\
\hline 2540 & \\
\hline
\end{tabular}

DMA operon5

\begin{tabular}{|l}
\hline 2672 \\
\hline Me-sinlge3 \\
\hline mtaA4 \\
\hline 6155 \\
\hline
\end{tabular}

Supplementary Figure 7 Methyltransferases in the genome of AMET1 and HMET1. mta, methanol-specific; mtm, methyamine-specific; $m$ tb, dimethylamine-specific; $m$ tt, trimethylamine-specific; pyl, methytransferase specific pyrrolysin synthesis. Shadowed: highly expressed; shadowed with dashed lines - moderately expressed. 
Halobacteriales

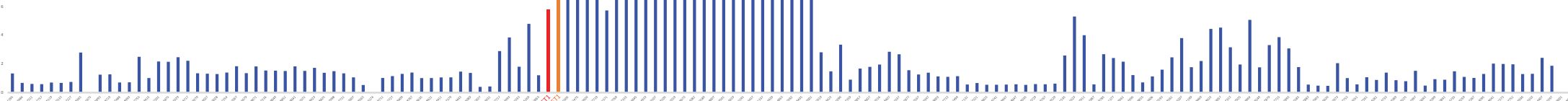

Supplementary Figure 8 UspA family distribution in archaea (based on arCOGs). The sum of number of UspA paralogs in 11 respective arCOGs is shown.

Genomes are shown approximately according to the archaeal taxonomy. No correction for genome size was implemented, but the effect for AMET1 and HMET1 will be even more pronounced if it would be applied. 


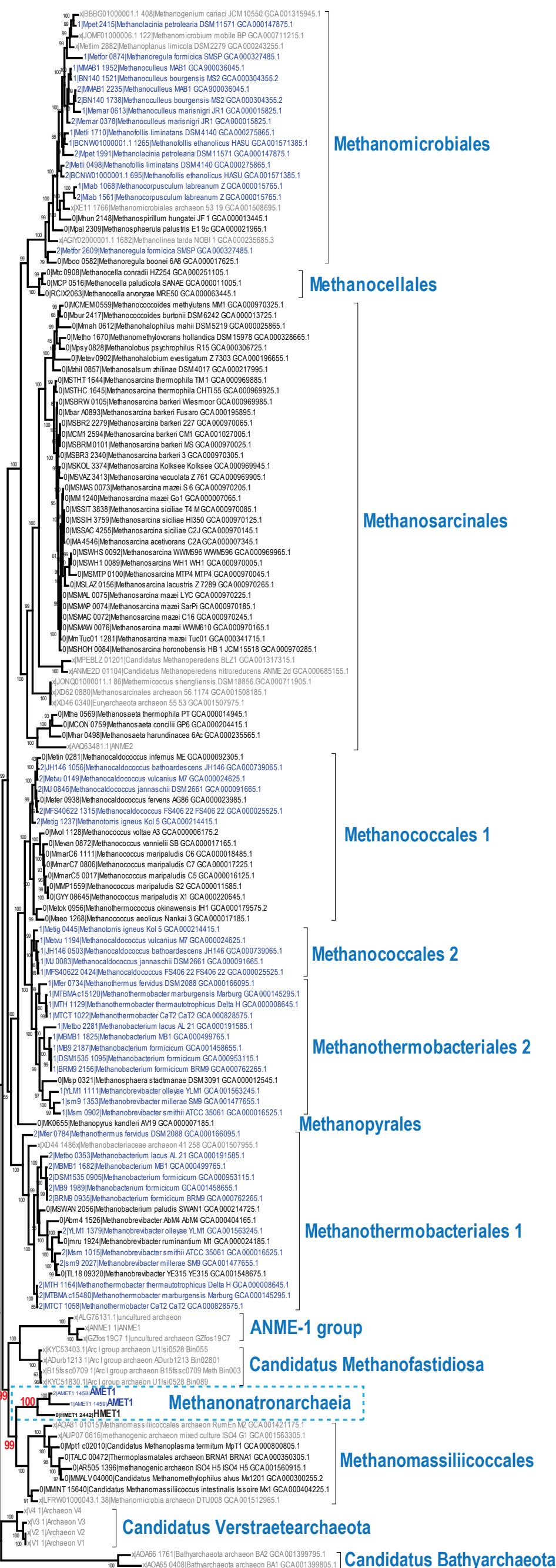

Supplementary Figure 9 Phylogeny of Methyl coenzyme M reductase alpha subunit (McrA). Branch support values calculated by PhyML are shown and for the clade containing AMET1 and HMET1 are colored red. The sequences are denoted by locus tag ID or genome partition ID, genome names and its code on the genbank FTP site. First part of the sequence name indicates the following: $x$ - incomplete genome (colored gray), 0 - one McrA gene in complete genomes (colored black), 1 - first copy of McrA and 2 - second copy of McrA (both colored blue). The original tree is available in Supplementary data 2. 


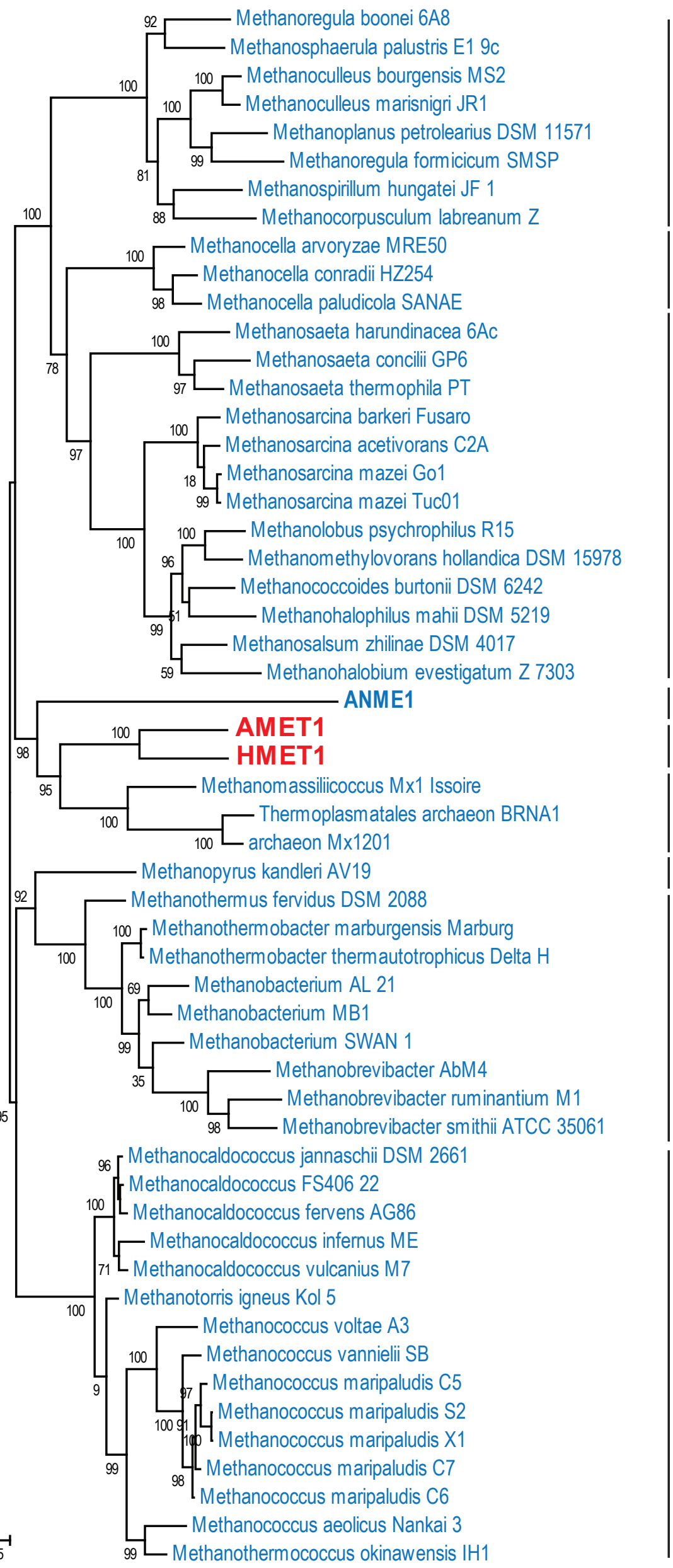

Methanomicrobiales

Methanocellales

Methanosarcinales

ANME1

Methanonatronarchaeia

Methanomassiliicoccales

Methanopyrales

Methanothermobacteriales

Methanococcales

Supplementary Figure 10 Phylogenetic tree of concatenated subunits of Mcr operon (FastTree method). The maximumlikelihood phylogenetic tree of the concatenated subunits of Methyl coenzyme M reductase complex (McrA, McrB, McrD and McrG) proteins was built using the FastTree program and includes 55 genomes and 1303 positions in the multiple alignment. Underlying multiple alignment can be provided by a request. 


\section{Supplementary Data 1. Original phylogenetic tree (PHYML method) of concatenated ribosomal proteins in Newick format schematically shown on the Figure 3A.}

The maximum-likelihood phylogenetic tree of the concatenated ribosomal proteins was built using the PHYML program as described in the Materials and Methods section. Underlying multiple alignment can be provided by a request.

The tree (below) is provided in Newick format, could be open in MEGA or in any other tree visualization software. To open the file copy the text below the dotted line to a text file with "tre" extension. MEGA (or other tree visualization software) should open the file automatically.

((Haladaptatus_cibarius_D43_GCA_000710615.1:0.05123227,(Haladaptatus_paucihalophilus_DX253_G CA_000376445.1:0.00194871,Haladaptatus_paucihalophilus_DX253_GCA_000187225.2:0.00018041)1.0 00000:0.04785972)1.000000:0.10199324,(Halovivax_ruber_XH_70_GCA_000328525.1:0.11364497,(Nat rialbaceae_archaeon_Tc_Br11_E2g1_GCA_001564255.1:0.08962486,((Natronococcus_occultus_SP4_GC A_000328685.1:0.05084899,(Natrialba_magadii_ATCC_43099_GCA_000025625.1:0.04625005,((Halopig er_xanaduensis_SH_6_GCA_000217715.1:0.03875813,(Natronobacterium_gregoryi_SP2_GCA_0002307 15.3:0.03871285,(Halobiforma_nitratireducens_JCM_10879_GCA_000337895.1:0.02463099,(Halobifor ma_lacisalsi_AJ5_GCA_000336655.1:0.00101970,Halobiforma_lacisalsi_AJ5_GCA_000226975.2:0.00056 931)1.000000:0.02176018)1.000000:0.01444914)1.000000:0.02089807)1.000000:0.01628374,(Natronol imnobius_innermongolicus_JCM_12255_GCA_000337215.1:0.04596348,(((Natronorubrum_tibetense_G A33_GCA_000383975.1:0.00129994,Natronorubrum_tibetense_GA33_GCA_000337235.1:0.00014338)1 .000000:0.04358080,(Natronorubrum_sulfidifaciens_JCM_14089_GCA_000337735.1:0.01612770,Natro norubrum_bangense_JCM_10635_GCA_000337715.1:0.01955264)1.000000:0.02400693)1.000000:0.01 739542,(Haloterrigena_turkmenica_DSM_5511_GCA_000025325.1:0.03255533,(Natrinema_pellirubru m_DSM_15624_GCA_000230735.3:0.02515212,Natrinema_J7_2_J7_2_GCA_000281695.1:0.03100661) 1.000000:0.02931047)1.000000:0.01135200)1.000000:0.01186265)1.000000:0.00753696)1.000000:0.0 0950184)1.000000:0.01470485)1.000000:0.02905290,(Natrialbaceae_archaeon_Tc_Br11_E2g14_GCA_0 01564275.1:0.09562658,Halostagnicola_larsenii_XH_48_GCA_000517625.1:0.07426316)1.000000:0.018 64931)1.000000:0.01456751)1.000000:0.01636741)1.000000:0.08007120,(((Halalkalicoccus_jeotgali_B3 _GCA_000196895.1:0.16303611,((Halococcus_saccharolyticus_DSM_5350_GCA_000336915.1:0.012092 25,(Halococcus_salifodinae_DSM_8989_GCA_000336935.1:0.02398395,Halococcus_agarilyticus_197A_ GCA_000334895.1:0.02019188)1.000000:0.01008883)1.000000:0.06461170,((Halococcus_hamelinensis _100A6_GCA_000336675.1:0.00071299,Halococcus_hamelinensis_100A6_GCA_000259215.1:0.000244 53)1.000000:0.09841495,(Halococcus_sediminicola_CBA1101_GCA_000755245.1:0.03553527,(Halococc us_thailandensis_JCM_13552_GCA_000336715.1:0.01096533,Halococcus_morrhuae_DSM_1307_GCA_ 000336695.1:0.01454966)1.000000:0.03520031)1.000000:0.03663527)1.000000:0.03199540)1.000000: 0.11074453)1.000000:0.03359312,(Salinarchaeum_Harcht_Bsk1_Harcht_Bsk1_GCA_000403645.1:0.195 52508,((Natronomonas_pharaonis_DSM_2160_GCA_000026045.1:0.06099901,Natronomonas_moolap ensis_8_8_11_GCA_000591055.1:0.07545333)1.000000:0.13377323,((Halapricum_salinum_CBA1105_G CA_000755225.1:0.10941697,(Halorhabdus_utahensis_DSM_12940_GCA_000023945.1:0.02671439,Hal orhabdus_tiamatea_SARL4B_GCA_000470655.1:0.02124075)1.000000:0.10358802)1.000000:0.0274276 0,(Halosimplex_carlsbadense_2_9_1_GCA_000337455.1:0.12351258,(Halomicrobium_mukohataei_DS M_12286_GCA_000023965.1:0.09788411,(Haloarcula_hispanica_N601_GCA_000504565.2:0.00080159, 
Haloarcula_hispanica_ATCC_33960_GCA_000223905.1:0.00183339)1.000000:0.09807337)1.000000:0.0 3589376)1.000000:0.02143987)1.000000:0.03124641)1.000000:0.02974697)1.000000:0.02325690)1.00 0000:0.01880577,((((HMET1:0.26590909,AMET1:0.26097624)1.000000:0.26077876,(((Archaeoglobus_p rofundus_DSM_5631_GCA_000025285.1:0.12510781,(Archaeoglobus_fulgidus_DSM_4304_GCA_00000 8665.1:0.15223232,((Ferroglobus_placidus_DSM_10642_GCA_000025505.1:0.11743320,(Geoglobus_ah angari_234_GCA_001006045.1:0.04125203,Geoglobus_acetivorans_SBH6_GCA_000789255.1:0.089471 27)1.000000:0.08682962)1.000000:0.04752603,(Archaeoglobus_sulfaticallidus_PM70_1_GCA_0003855 65.1:0.20447175,Archaeoglobus_veneficus_SNP6_GCA_000194625.1:0.10810310)1.000000:0.04734308 )0.999531:0.03093245)1.000000:0.03564157)1.000000:0.31574834,((((Aciduliprofundum_boonei_T469 _GCA_000025665.1:0.05038879,Aciduliprofundum_MAR08_339_MAR08_339_GCA_000327505.1:0.061 38455)1.000000:0.25571818,(Thermoplasma_volcanium_GSS1_GCA_000011185.1:0.27474882,((Picrop hilus_torridus_DSM_9790_GCA_000008265.1:0.15868661,(Ferroplasma_acidarmanus_fer1_GCA_00015 2265.2:0.17265623,(Acidiplasma_aeolicum_VT_GCA_001402945.1:0.00214505,(Acidiplasma_MBA_1_M BA_1_GCA_000949015.1:0.00025867,(Acidiplasma_cupricumulans_JCM_13668_GCA_001316225.1:0.02 945152,Acidiplasma_aeolicum_V_GCA_001399695.1:8.75780890042492e-

07)0.000000:6.79949471880265e-

07)0.599352:0.00022420)1.000000:0.11846571)1.000000:0.08548672)1.000000:0.15028061,(Thermogy mnomonas_acidicola_JCM_13583_GCA_001316105.1:0.29707007,(Thermoplasmatales_archaeon_E_pl asma_GCA_000496135.1:0.31865587,(uncultured_archaeon_GCA_000965745.1:0.24265394,Thermopla smatales_archaeon_A_plasma_GCA_000447225.1:0.22686035)0.999998:0.03719285)1.000000:0.02839 825)1.000000:0.03348651)1.000000:0.05935084)1.000000:0.35431395)1.000000:0.09531179,(uncultur ed_marine_group_II_euryarchaeote_GCA_000246735.1:0.75033593,((Methanomicrobia_archaeon_DT U008_GCA_001512965.1:0.15445719,Candidatus_Methanomassiliicoccus_intestinalis_Issoire_Mx1_GC A_000404225.1:0.16342023)1.000000:0.13174102,(Methanomassiliicoccales_archaeon_RumEn_M2_GC A_001421175.1:0.18496268,(methanogenic_archaeon_mixed_culture_ISO4_G1_GCA_001563305.1:0.1 3641264,(Candidatus_Methanoplasma_termitum_MpT1_GCA_000800805.1:0.13252907,(Thermoplasm atales_archaeon_BRNA1_BRNA1_GCA_000350305.1:0.11852568,(methanogenic_archaeon_ISO4_H5_IS O4_H5_GCA_001560915.1:0.09803630,Candidatus_Methanomethylophilus_alvus_Mx1201_GCA_00030 0255.2:0.07047496)0.515303:0.02210804)1.000000:0.11056534)1.000000:0.03270439)1.000000:0.066 51469)1.000000:0.15961075)1.000000:0.16387830)1.000000:0.08734484)1.000000:0.13591136,((((Pala eococcus_pacificus_DY20341_GCA_000725425.1:0.09904985,(Thermococcus_2319x1_2319x1_GCA_00 1484685.1:0.06276820,(((Thermococcus_onnurineus_NA1_GCA_000018365.1:0.02461438,(Thermococc us_cleftensis_CL1_GCA_000265525.1:0.01324934,Thermococcus_4557_4557_GCA_000221185.1:0.032 81204)1.000000:0.01426398)1.000000:0.01425800,(Thermococcus_kodakarensis_KOD1_GCA_0000099 65.1:0.02886184,((Thermococcus_nautili_30_1_GCA_000585495.1:0.01315708,Thermococcus_euryther malis_A501_GCA_000769655.1:0.01596021)1.000000:0.00696172,(Thermococcus_guaymasensis_DSM _11113_GCA_000816105.1:0.01824294,(Thermococcus_gammatolerans_EJ3_GCA_000022365.1:0.0123 7558,Thermococcus_AM4_AM4_GCA_000151205.2:0.01030295)1.000000:0.01102703)1.000000:0.0090 8310)1.000000:0.01529137)1.000000:0.02029325)1.000000:0.05832865,((Pyrococcus_yayanosii_CH1_ GCA_000215995.1:0.02637222,((Pyrococcus_furiosus_DSM_3638_GCA_000007305.1:0.00066118,Pyroc occus_furiosus_COM1_GCA_000275605.1:7.66176136462553e-

07)1.000000:0.02626733,(Pyrococcus_NA2_NA2_GCA_000211475.1:0.03051424,(Pyrococcus_ST04_ST0 4_GCA_000263735.1:0.01952884,Pyrococcus_NCB100_NCB100_GCA_001577775.1:0.01332909)1.0000 00:0.00687595)1.000000:0.00810774)1.000000:0.02596486)1.000000:0.06690865,(Thermococcus_para 
Ivinellae_ES1_GCA_000517445.1:0.01197970,(Thermococcus_barophilus_MP_GCA_000151105.2:0.001 55502,Thermococcus_barophilus_CH5_GCA_001433455.1:0.00110078)1.000000:0.01083654)1.000000: 0.03022989)1.000000:0.01607211)1.000000:0.02046989)1.000000:0.03370048)1.000000:0.30010010,(( Methanocaldococcus_infernus_ME_GCA_000092305.1:0.09901460,(Methanocaldococcus_vulcanius_M 7_GCA_000024625.1:0.05203855,(Methanocaldococcus_jannaschii_DSM_2661_GCA_000091665.1:0.00 611354,(Methanocaldococcus_FS406_22_FS406_22_GCA_000025525.1:0.00788400,(Methanocaldococ cus_fervens_AG86_GCA_000023985.1:0.02263590,Methanocaldococcus_bathoardescens_JH146_GCA_ 000739065.1:0.01269380)1.000000:0.00890787)1.000000:0.00500892)1.000000:0.01432220)1.000000: 0.03842180)1.000000:0.09376561,(Methanotorris_igneus_Kol_5_GCA_000214415.1:0.07233138,((Met hanothermococcus_okinawensis_IH1_GCA_000179575.2:0.06350673,Methanococcus_aeolicus_Nankai _3_GCA_000017185.1:0.13607815)1.000000:0.05344917,(Methanococcus_voltae_A3_GCA_000006175. 2:0.13233320,(Methanococcus_vannielii_SB_GCA_000017165.1:0.06854276,((Methanococcus_maripal udis_X1_GCA_000220645.1:0.00059222,Methanococcus_maripaludis_S2_GCA_000011585.1:0.0009395 4)1.000000:0.01532744,(Methanococcus_maripaludis_C5_GCA_000016125.1:0.01196337,(Methanococ cus_maripaludis_C7_GCA_000017225.1:0.00519695,Methanococcus_maripaludis_C6_GCA_000018485. 1:0.00638043)1.000000:0.00943218)1.000000:0.00887549)1.000000:0.04508361)1.000000:0.06183411 1.000000:0.09289445)1.000000:0.13845807)1.000000:0.05921718)1.000000:0.25510728)1.000000:0.0 5443293,(Hadesarchaea_archaeon_YNP_N21_GCA_001515215.1:0.56143812,(Methanopyrus_kandleri_ AV19_GCA_000007185.1:0.39905541,(Methanothermus_fervidus_DSM_2088_GCA_000166095.1:0.173 34676,(Methanobacteriaceae_archaeon_41_258_GCA_001507955.1:0.13084187,(((Methanobrevibacte r_AbM4_AbM4_GCA_000404165.1:0.13293386,((Methanobrevibacter_ruminantium_M1_GCA_000024 185.1:0.03672111,Methanobrevibacter_olleyae_YLM1_GCA_001563245.1:0.03896783)1.000000:0.1009 7759,(Methanobrevibacter_smithii_ATCC_35061_GCA_000016525.1:0.05430356,Methanobrevibacter_ millerae_SM9_GCA_001477655.1:0.05302039)1.000000:0.09524353)0.999952:0.02863825)1.000000:0. 13065595,(Methanosphaera_stadtmanae_DSM_3091_GCA_000012545.1:0.27038885,(Methanobacteri um_formicicum_BRM9_GCA_000762265.1:0.12499215,(Methanobacterium_paludis_SWAN1_GCA_000 214725.1:0.10007107,Methanobacterium_lacus_AL_21_GCA_000191585.1:0.10673631)1.000000:0.046 56116)1.000000:0.04713163)0.999998:0.04752644)1.000000:0.06680411,(Methanothermobacter_ther mautotrophicus_Delta_H_GCA_000008645.1:0.02002038,(Methanothermobacter_marburgensis_Marb urg_GCA_000145295.1:0.01333629,Methanothermobacter_CaT2_CaT2_GCA_000828575.1:0.00575121 )1.000000:0.01313801)1.000000:0.09103260)1.000000:0.05935742)1.000000:0.10854142)1.000000:0.1 7852959)1.000000:0.03679858)1.000000:0.02740279)1.000000:0.03608600,(((((miscellaneous_Crenarc haeota_group_6_archaeon_AD8_1_GCA_001273385.1:0.37591586,(Bathyarchaeota_archaeon_BA2_GC A_001399795.1:0.14545912,Bathyarchaeota_archaeon_BA1_GCA_001399805.1:0.16450952)1.000000: 0.12912518)1.000000:0.21932828,(Candidatus_Caldiarchaeum_subterraneum_GCA_000270325.1:0.67 884245,(((Thaumarchaeota_archaeon_CSP1_1_GCA_001443365.1:0.14533943,((Candidatus_Nitrosopel agicus_brevis_CN25_GCA_000812185.1:0.23695796,(Thaumarchaeota_archaeon_SCGC_AAA007_023_ SCGC_AAA007_O23_GCA_000402075.1:0.01974660,Marine_Group_Itthaumarchaeote_SCGC_AB_629_I 23_SCGC_AB_629_123_GCA_000399765.1:0.01405088)1.000000:0.17529252)1.000000:0.04792613,(Ce narchaeum_symbiosum_A_GCA_000200715.1:0.27059035,((Candidatus_Nitrosoarchaeum_limnia_BG2 0_GCA_000241145.2:0.03873322,Candidatus_Nitrosoarchaeum_koreensis_MY1_GCA_000220175.2:0.0 3856796)1.000000:0.06796443,((Thaumarchaeota_archaeon_casp_thauma3_GCA_001510275.1:0.0756 3713,(Thaumarchaeota_archaeon_casp_thauma1_GCA_001510225.1:0.13392910,Candidatus_Nitrosop umilus_AR2_AR2_GCA_000299395.1:0.05791408)1.000000:0.01855937)1.000000:0.01363008,(Candida 
tus_Nitrosopumilus_koreensis_AR1_GCA_000299365.1:0.01950340,(Nitrosopumilus_maritimus_SCM1_ GCA_000018465.1:0.01673884,(Marine_Group_I_thaumarchaeote_SCGC_RSA3_GCA_000746745.1:0.0 0253396,Marine_Group_I_thaumarchaeote_SCGC_AAA799_E16_GCA_000724145.1:0.00060928)1.0000 00:0.01550460)1.000000:0.01333835)1.000000:0.04287605)1.000000:0.03638214)1.000000:0.0811087 9)1.000000:0.04696913)1.000000:0.02695169)1.000000:0.04492101,(Thaumarchaeota_archaeon_MY2 _MY2_GCA_000685395.1:0.08749905,(Thaumarchaeota_archaeon_SAT1_SAT1_GCA_000955905.3:0.09 049932,Thaumarchaeota_archaeon_N4_N4_GCA_000723185.1:0.08586084)0.999143:0.02701920)1.00 0000:0.10475525)1.000000:0.27538537,(Thaumarchaeota_archaeon_MY3_MY3_GCA_000802205.2:0.2 6895286,(Candidatus_Nitrososphaera_gargensis_Ga9_2_GCA_000303155.1:0.09082603,(Nitrososphaer a_viennensis_EN76_GCA_000698785.1:0.01559267,Candidatus_Nitrososphaera_evergladensis_SR1_GC A_000730285.1:0.01463134)1.000000:0.09157964)1.000000:0.11253920)1.000000:0.18296798)1.0000 00:0.41980146)1.000000:0.09590949)1.000000:0.07844507,(Candidatus_Korarchaeum_cryptofilum_OP F8_GCA_000019605.1:0.76782167,(((Sulfolobales_archaeon_Acd1_GCA_000389735.1:0.22425471,((Sulf olobus_tokodaii_7_GCA_000011205.1:0.12598142,(Sulfolobus_acidocaldarius_SUSAZ_GCA_000508305. 1:0.00829700,(Sulfolobus_acidocaldarius_DSM_639_GCA_000012285.1:9.01554252415256e07,(Sulfolobus_acidocaldarius_N8_GCA_000340315.1:0.00025543,Sulfolobus_acidocaldarius_Ron12_I_ GCA_000338775.1:7.52127849373203e-07)0.000000:6.13439542072721e-

07)0.999971:0.00254216)1.000000:0.18156581)1.000000:0.06802457,(((Sulfolobus_solfataricus_P2_GC A_000007005.1:0.00038150,Sulfolobus_solfataricus_98_2_GCA_000024745.1:7.45153505423486e07)1.000000:0.02434353,(Sulfolobus_islandicus_M_16_27_GCA_000022425.1:0.00040048,(((Sulfolobus _islandicus_M_14_25_GCA_000022405.1:0.00203694,Sulfolobus_islandicus_M_16_4_GCA_000022445. 1:0.00025451)0.990253:0.00014583,(Sulfolobus_islandicus_L_S_2_15_GCA_000022385.1:0.00063530,( Sulfolobus_islandicus_Y_N_15_51_GCA_000022485.1:0.00127039,Sulfolobus_islandicus_Y_G_57_14_G CA_000022465.1:0.00038102)0.999910:0.00025345)1.000000:0.00201680)1.000000:0.00063549,(Sulfol obus_islandicus_LAL14_1_GCA_000364745.1:0.00038102,(Sulfolobus_islandicus_REY15A_GCA_000189 555.1:0.00038121,Sulfolobus_islandicus_HVE10_4_GCA_000189575.1:0.00025410)0.000000:9.5529349 2758942e-07)1.000000:0.00087074)0.000000:6.68946732554598e-

07)1.000000:0.03078770)1.000000:0.17956311,(Acidianus_hospitalis_W1_GCA_000213215.1:0.161704 21,(Sulfolobales_archaeon_AZ1_GCA_000565255.1:0.16908336,(Metallosphaera_sedula_DSM_5348_G CA_000016605.1:0.07871228,Metallosphaera_cuprina_Ar_4_GCA_000204925.1:0.11004420)1.000000: 0.17606086)1.000000:0.03729620)1.000000:0.06740331)1.000000:0.03649271)1.000000:0.05679829)1 .000000:0.28128659,(Ignisphaera_aggregans_DSM_17230_GCA_000145985.1:0.46539319,(((Ignicoccus _hospitalis_KIN4_I_GCA_000017945.1:0.35189916,(Pyrolobus_fumarii_1A_GCA_000223395.1:0.130792 51,(Pyrodictium_delaneyi_Su06_GCA_001412615.1:0.07801522,Hyperthermus_butylicus_DSM_5456_G CA_000015145.1:0.05412387)1.000000:0.11292250)1.000000:0.10949388)1.000000:0.04542171,(Fervi dicoccus_fontis_Kam940_GCA_000258425.1:0.45907557,((Aeropyrum_pernix_K1_GCA_000011125.1:0. 02445126,Aeropyrum_camini_SY1_JCM_12091_GCA_000591035.1:0.03171795)1.000000:0.24423608,( Caldisphaera_lagunensis_DSM_15908_GCA_000317795.1:0.27663897,Acidilobus_saccharovorans_345_ 15_GCA_000144915.1:0.25194656)1.000000:0.18962185)1.000000:0.13281033)1.000000:0.04173816)0 .999823:0.03314826,(Staphylothermus_hellenicus_DSM_12710_GCA_000092465.1:0.17378991,(Therm ogladius_cellulolyticus_1633_GCA_000264495.1:0.20582602,(Thermosphaera_aggregans_DSM_11486_ GCA_000092185.1:0.15635189,(Desulfurococcus_mucosus_DSM_2162_GCA_000186365.1:0.07307443, (Desulfurococcus_kamchatkensis_1221n_GCA_000020905.1:0.01315008,Desulfurococcus_fermentans_ DSM_16532_GCA_000231015.3:0.00977410)1.000000:0.07433146)1.000000:0.08938098)1.000000:0.0 
7553973)1.000000:0.07011047)1.000000:0.14775840)0.997541:0.03639137)1.000000:0.05101520)1.00 0000:0.11869037,((crenarchaeote_JGI_OTU_1_JGI_OTU_1_GCA_000494205.1:0.00043023,Crenarchaeo ta_archaeon_SCGC_AAA471_B05_SCGC_AAA471_B05_GCA_000380705.1:9.52064435143598e07)1.000000:0.69068221,(Thermofilum_pendens_Hrk_5_GCA_000015225.1:0.43198513,(((Pyrobaculu m_islandicum_DSM_4184_GCA_000015205.1:0.06049385,((Pyrobaculum_oguniense_TE7_GCA_000247 545.1:0.00895138,Pyrobaculum_arsenaticum_DSM_13514_GCA_000016385.1:0.00904370)1.000000:0. 08264446,(Pyrobaculum_neutrophilum_V24Sta_GCA_000019805.1:0.06766760,Pyrobaculum_WP30_W P30_GCA_001189275.1:0.07880506)1.000000:0.02116796)1.000000:0.02129806)1.000000:0.12861275, (Thermoproteus_uzoniensis_768_20_GCA_000193375.1:0.09416815,Thermoproteus_tenax_Kra_1_GCA _000253055.1:0.10665605)1.000000:0.12320705)1.000000:0.14826591,(Vulcanisaeta_distributa_DSM_ 14429_GCA_000148385.1:0.23069950,Caldivirga_maquilingensis_IC_167_GCA_000018305.1:0.3737007 2)1.000000:0.08087806)1.000000:0.21983773)1.000000:0.08833119)1.000000:0.05605042)1.000000:0. 07267807)1.000000:0.05856797)1.000000:0.05306699,(Thorarchaeota_archaeon_SMTZ1_83_GCA_001 563325.1:0.58862035,Lokiarchaeum_GC14_75_GCA_000986845.1:0.78944285)1.000000:0.11740830)1. 000000:0.08424858,(((archaeon_GW2011_AR5_GCA_000806115.1:0.74610207,(Candidatus_Nanosalina rum_J07AB56_GCA_000220355.1:0.38355070,(Nanohaloarchaea_archaeon_B1_Br10_U2g1_GCA_0015 63875.1:0.21368000,Candidatus_Haloredivivus_G17_G17_GCA_000236195.2:0.27207799)0.999996:0.0 6502123)1.000000:0.61378983)1.000000:0.10665170,((nanoarchaeote_Nst1_GCA_000387965.1:0.6478 9428,Nanoarchaeum_equitans_Kin4_M_GCA_000008085.1:0.43034450)1.000000:0.28552108,(archaeo n_GW2011_AR15_GCA_000830295.1:0.56207839,(archaeon_GW2011_AR17_GCA_000805995.1:0.5494 9369,(archaeon_GW2011_AR20_GCA_000830315.1:0.44916639,(Nanoarchaeota_archaeon_SCGC_AAA 011_G17_SCGC_AAA011_G17_GCA_000402515.1:0.00055146,(Nanoarchaeota_archaeon_SCGC_AAA01 1_L22_SCGC_AAA011_L22_GCA_000380905.1:9.38692098142289e-

07,Nanoarchaeota_archaeon_JGI_OTU_1_JGI_OTU_1_GCA_000494105.1:8.04941246397664e07)0.667912:0.00042005)1.000000:0.44042390)1.000000:0.06781675)1.000000:0.07176073)1.000000: 0.16821464)1.000000:0.09945572)0.976791:0.03863879,(Candidatus_Micrarchaeum_acidiphilum_ARM AN_2:1.07243840,(archaeon_GW2011_AR10_GCA_000830275.1:0.35425844,Candidatus_lainarchaeum _andersonii_SCGC_AAA011_E11_GCA_000402355.1:0.39370488)1.000000:0.45543242)1.000000:0.139 84988)1.000000:0.07128923)1.000000:0.05172813)1.000000:0.07458726)1.000000:0.05944858)1.0000 00:0.07011384,(((Methanomicrobiales_archaeon_53_19_GCA_001508695.1:0.18528110,Methanocorpu sculum_labreanum_Z_GCA_000015765.1:0.25287978)1.000000:0.10429709,(((Methanospirillum_hunga tei_JF_1_GCA_000013445.1:0.28398701,((Methanolinea_tarda_NOBI_1_GCA_000235685.3:0.15121338 ,Methanolinea_SDB_GCA_001412335.1:0.11412283)1.000000:0.07353717,(Methanosphaerula_palustri S_E1_9c_GCA_000021965.1:0.20600831,(Methanoregula_formicica_SMSP_GCA_000327485.1:0.099887 90,Methanoregula_boonei_6A8_GCA_000017625.1:0.10423915)1.000000:0.10816499)0.998157:0.0316 6132)1.000000:0.03234927)1.000000:0.04353559,(Methanofollis_liminatans_DSM_4140_GCA_000275 865.1:0.07864542,Methanofollis_ethanolicus_HASU_GCA_001571385.1:0.09868813)1.000000:0.15829 358)0.989533:0.02268906,((Methanogenium_cariaci_JCM_10550_GCA_001315945.1:0.19025125,(Met hanomicrobium_mobile_BP_GCA_000711215.1:0.16606478,(Methanoplanus_limicola_DSM_2279_GCA _000243255.1:0.11738222,Methanolacinia_petrolearia_DSM_11571_GCA_000147875.1:0.12023825)0. 999999:0.03057445)1.000000:0.05143994)1.000000:0.09481733,(Methanoculleus_marisnigri_JR1_GCA _000015825.1:0.06600606,Methanoculleus_bourgensis_MS2_GCA_000304355.2:0.06457032)1.000000: 0.14920374)1.000000:0.02812752)1.000000:0.03345833)1.000000:0.29593412,((Methanocella_paludic ola_SANAE_GCA_000011005.1:0.09162871,Methanocella_conradii_HZ254_GCA_000251105.1:0.052466 
35)1.000000:0.36421078,((Candidatus_Methanoperedens_nitroreducens_ANME_2d_GCA_000685155.1 :0.34705411,(((Methanosarcina_barkeri_3_GCA_000970305.1:0.01580061,((Methanosarcina_vacuolata _Z_761_GCA_000969905.1:0.00067275,Methanosarcina_Kolksee_Kolksee_GCA_000969945.1:0.000225 31)1.000000:0.01272098,(Methanosarcina_barkeri_Fusaro_GCA_000195895.1:0.01251839,Methanosar cina_barkeri_CM1_GCA_001027005.1:0.01283474)1.000000:0.01094662)1.000000:0.01002961)1.00000 0:0.03120922,(Methanosarcina_lacustris_Z_7289_GCA_000970265.1:0.05447957,(Methanosarcina_ace tivorans_C2A_GCA_000007345.1:0.02806603,(Methanosarcina_horonobensis_HB_1_JCM_15518_GCA_ 000970285.1:0.01487846,(Methanosarcina_mazei_LYC_GCA_000970225.1:0.00056965,(Methanosarcin a_mazei_S_6_GCA_000970205.1:0.00051159,(Methanosarcina_mazei_SarPi_GCA_000970185.1:0.0007 1202,(Methanosarcina_mazei_WWM610_GCA_000970165.1:0.00045800,Methanosarcina_mazei_C16_ GCA_000970245.1:0.00055577)0.999998:0.00037964)1.000000:0.00055312)0.999992:0.00070182)1.00 0000:0.02226322)1.000000:0.01072431)1.000000:0.01247846)1.000000:0.01909048)1.000000:0.19168 022,(((Methanomethylovorans_hollandica_DSM_15978_GCA_000328665.1:0.14771835,Methanolobus_ psychrophilus_R15_GCA_000306725.1:0.13972290)1.000000:0.06414832,(Methanohalophilus_mahii_D SM_5219_GCA_000025865.1:0.20571918,Methanococcoides_burtonii_DSM_6242_GCA_000013725.1:0 .14784513)1.000000:0.03252925)1.000000:0.02786006,(Methanohalobium_evestigatum_Z_7303_GCA _000196655.1:0.24789342,Methanosalsum_zhilinae_DSM_4017_GCA_000217995.1:0.17280441)1.0000 00:0.03847391)1.000000:0.05047643)1.000000:0.14543294)1.000000:0.06386940,(Methanosaeta_ther mophila_PT_GCA_000014945.1:0.37159493,(Methanosarcinales_archeaon_56_1174_GCA_001508185. 1:7.47330233004044e-

07,(Methermicoccus_shengliensis_DSM_18856_GCA_000711905.1:0.00205220,Euryarchaeota_archaeo n_55_53_GCA_001507975.1:9.66200075269519e-

07)0.999833:0.00081972)1.000000:0.37992425)1.000000:0.05353189)1.000000:0.04193022)1.000000: 0.04490705)1.000000:0.06617083)1.000000:0.10318645)1.000000:0.45930623,((((uncultured_archaeo n_A07HN63_GCA_000496215.1:0.02308102,Halonotius_J07HN6_GCA_000416025.1:0.00272320)1.0000 00:0.17007836,(haloarchaeon_3A1_DGR_3A1_DGR_GCA_000447865.2:0.07159277,(Halopenitus_DYS4 _DYS4_GCA_001462205.1:0.06467871,(uncultured_archaeon_A07HR67_GCA_000496175.1:0.05711499 ,Halorubrum_lacusprofundi_ATCC_49239_GCA_000022205.1:0.04643598)1.000000:0.06092600)1.0000 00:0.02076445)1.000000:0.05228200)1.000000:0.03330180,(Candidatus_Halobonum_tyrrellensis_G22_ GCA_000495475.1:0.13700748,(halophilic_archaeon_DL31_DL31_GCA_000224475.1:0.07415673,(Halol amina_sediminis_halo7_GCA_001282785.1:0.02876453,(Halolamina_rubra_CBA1107_GCA_000739555. 1:0.01149814,Halolamina_pelagica_CDK2_GCA_001307315.1:0.02466442)1.000000:0.01767003)1.0000 00:0.03599326)1.000000:0.09608803)1.000000:0.02837263)1.000000:0.01604173,((uncultured_archae on_A07HB70_GCA_000496195.1:0.20022254,Haloplanus_natans_DSM_17983_GCA_000427685.1:0.08 982940)1.000000:0.03212121,((Halogranum_salarium_B_1_GCA_000283335.1:0.09543084,Halobacteri aceae_archaeon_SB9_SB9_GCA_001469955.1:0.10331274)1.000000:0.03415919,((Haloferax_volcanii_D S2_GCA_000025685.1:0.02571660,Haloferax_mediterranei_ATCC_33500_GCA_000306765.2:0.0247827 3)1.000000:0.08676013,(Halogeometricum_borinquense_DSM_11551_GCA_000172995.2:0.06798800,( Halobellus_rufus_CBA1103_GCA_000739575.1:0.05201178,(Haloquadratum_walsbyi_DSM_16790_GCA _000009185.1:0.00045087,Haloquadratum_walsbyi_C23_GCA_000237865.1:0.00223657)1.000000:0.12 983118)1.000000:0.04410964)1.000000:0.02880679)1.000000:0.02650112)1.000000:0.02611597)1.000 000:0.01874596)1.000000:0.04425391)0.925355:0.02340424,((Halarchaeum_acidiphilum_MH1_52_1_ GCA_000474235.1:0.00086993,Halarchaeum_acidiphilum_MH1_52_1_GCA_000400975.1:0.00525486)1 .000000:0.12519878,((Halanaeroarchaeum_sulfurireducens_M27_SA2_GCA_001305655.1:0.00156821, 
Halanaeroarchaeum_sulfurireducens_HSR2_GCA_001011115.1:5.19095309231714e-

07)1.000000:0.16062930,((Halobacterium_salinarum_R1_GCA_000069025.1:0.00026104,Halobacterium _salinarum_NRC_1_GCA_000006805.1:0.00026500)1.000000:0.09247413,(Halobacterium_hubeiense_J 20_1_GCA_001488575.1:0.05224023,Halobacterium_DL1_DL1_GCA_000230955.3:0.06218804)1.00000 0:0.02412650)1.000000:0.05864945)0.999993:0.02730534)1.000000:0.06969867)1.000000:0.01962589 )1.000000:0.02941992); 
Supplementary Data 2. Original phylogenetic tree of McrA subunit (PHYML method) in Newick format shown on the Supplementary Figure 9.

The maximum-likelihood phylogenetic tree of the McrA proteins was built using the PHYML program as described in the Materials and Methods section. Underlying multiple alignment can be provided by a request.

The tree (below) is provided in Newick format, could be open in MEGA or in any other tree visualization software. To open the file copy the text below the dotted line to a text file with "tre" extension. MEGA (or other tree visualization software) should open the file automatically.

(Metin_0281|Methanocaldococcus_infernus_ME_GCA_000092305.1|1003473330:0.02751309,JH146_1 056|Methanocaldococcus_bathoardescens_JH146_GCA_000739065.1|1009883047:0.01382378,(Metvu _0149|Methanocaldococcus_vulcanius_M7_GCA_000024625.1|1002866825:0.00191529,((Metig_1237 | Methanotorris_igneus_Kol_5_GCA_000214415.1|1005029408:0.02604484,(((Metig_0445|Methanoto rris_igneus_Kol_5_GCA_000214415.1|1005028635:0.03087334,(Metvu_1194|Methanocaldococcus_vul canius_M7_GCA_000024625.1|1002867860:0.01455030,(JH146_0503|Methanocaldococcus_bathoarde scens_JH146_GCA_000739065.1|1009882501:0.01065102,(MJ_0083|Methanocaldococcus_jannaschii_ DSM_2661_GCA_000091665.1|1003420464:0.00334200,MFS40622_0424|Methanocaldococcus_FS406 _22_FS406_22_GCA_000025525.1|1003010328:0.00441005)0.998355:0.00553570)0.985922:0.0109641 0)1.000000:0.02529925)0.999723:0.02344265,((Mfer_0734|Methanothermus_fervidus_DSM_2088_GC A_000166095.1|1003993923:0.06983857,(MTBMA_c15120|Methanothermobacter_marburgensis_Mar burg_GCA_000145295.1|1003678604:0.00468987,(MTH_1129|Methanothermobacter_thermautotrop hicus_Delta_H_GCA_000008645.1|1000281338:9.80022079580978e-

07,MTCT_1022|Methanothermobacter_CaT2_CaT2_GCA_000828575.1|1011387870:0.00188642)0.992 979:0.00482651)1.000000:0.06949969)0.999997:0.03227802,((Metbo_2281|Methanobacterium_lacus_ AL_21_GCA_000191585.1|1004476749:0.11454996,(MBMB1_1825|Methanobacterium_MB1_GCA_00 0499765.1|1008479935:0.01104366,(MB9_2187|Methanobacterium_formicicum_GCA_001458655.1|1 015170296:6.72892943884776e-

07,(DSM1535_1095|Methanobacterium_formicicum_GCA_000953115.1|1012186986:0.00186765,BRM 9_2156|Methanobacterium_formicicum_BRM9_GCA_000762265.1|1010556959:7.51808601476544e07)0.000000:9.81892455737642e-

07)0.999918:0.01654393)1.000000:0.05356483)1.000000:0.04822860,(Msp_0321|Methanosphaera_st adtmanae_DSM_3091_GCA_000012545.1|1000868365:0.14060807,(YLM1_1111|Methanobrevibacter_ olleyae_YLM1_GCA_001563245.1|1016364553:0.07972982,(sm9_1353|Methanobrevibacter_millerae_ SM9_GCA_001477655.1|1015276403:0.05787367,Msm_0902|Methanobrevibacter_smithii_ATCC_350 61_GCA_000016525.1|1001555353:0.06257526)0.999999:0.04110549)1.000000:0.07694601)0.977224: 0.03119727)0.999467:0.03513120)1.000000:0.04686394)1.000000:0.07083314,(MK0655|Methanopyru s_kandleri_AV19_GCA_000007185.1|1000090767:0.18124921,((Mfer_0784|Methanothermus_fervidus _DSM_2088_GCA_000166095.1|1003993973:0.09493235,(XD44_1486x|Methanobacteriaceae_archaeo n_41_258_GCA_001507955.1|1115316079:0.07457850,(((Metbo_0353|Methanobacterium_lacus_AL_ 21_GCA_000191585.1|1004474859:0.06509425,(MBMB1_1682|Methanobacterium_MB1_GCA_00049 9765.1|1008479804:0.00709666,(DSM1535_0905|Methanobacterium_formicicum_GCA_000953115.1| 1012186808:7.05194082058862e- 
07,(MB9_1989|Methanobacterium_formicicum_GCA_001458655.1|1015170110:9.61188115364321e07,BRM9_0935|Methanobacterium_formicicum_BRM9_GCA_000762265.1|1010555753:5.7365775704 1058e-07)0.000000:9.51208332080561e-

07)0.999619:0.00972434)1.000000:0.05206346)1.000000:0.03532760,(MSWAN_2056|Methanobacteri um_paludis_SWAN1_GCA_000214725.1|1005042534:0.05505182,(Abm4_1526|Methanobrevibacter_A bM4_AbM4_GCA_000404165.1|1007743827:0.10766585,((YLM1_1379|Methanobrevibacter_olleyae_Y LM1_GCA_001563245.1|1016364821:0.02054417,mru_1924|Methanobrevibacter_ruminantium_M1_ GCA_000024185.1|1002801036:0.03336500)1.000000:0.06886985,(Msm_1015|Methanobrevibacter_s mithii_ATCC_35061_GCA_000016525.1|1001555466:0.05360302,(sm9_2027|Methanobrevibacter_mill erae_SM9_GCA_001477655.1|1015277052:0.01706308,TL18_09320|Methanobrevibacter_YE315_YE31 5_GCA_001548675.1|1016086870:0.02944717)0.999034:0.01424809)1.000000:0.03033290)1.000000:0 .04871457)1.000000:0.08423282)0.584826:0.01649912)0.999992:0.03673261,(MTH_1164|Methanothe rmobacter_thermautotrophicus_Delta_H_GCA_000008645.1|1000281373:0.00191846,(MTBMA_c1548 0|Methanothermobacter_marburgensis_Marburg_GCA_000145295.1|1003678640:0.00378640,MTCT_ 1058|Methanothermobacter_CaT2_CaT2_GCA_000828575.1|1011387906:0.00188925)0.850235:0.001 85755)1.000000:0.02947319)1.000000:0.05691291)1.000000:0.05933491)1.000000:0.12240718,((((AA Q63481.1|ANME2|34305116:0.28989388,(Mhar_0498|Methanosaeta_harundinacea_6Ac_GCA_00023 5565.1|1005532317:0.10707400,(MCON_0759|Methanosaeta_concilii_GP6_GCA_000204415.1|10048 25313:0.09658198,Mthe_0569|Methanosaeta_thermophila_PT_GCA_000014945.1|1001273562:0.041 86360)0.935463:0.02379401)1.000000:0.13007466)1.000000:0.10563715,((XD46_0340|Euryarchaeota_ archaeon_55_53_GCA_001507975.1|1115318852:5.32011406957467e-

07,(XD62_0880|Methanosarcinales_archeaon_56_1174_GCA_001508185.1|1115337013:9.3758773408 708 e-

07,JONQ01000011.1_86|Methermicoccus_shengliensis_DSM_18856_GCA_000711905.1|1502897550:7 .63461312041489e-07)0.000000:6.80528675933942e-

07)1.000000:0.18500600,((ANME2D_01104|Candidatus_Methanoperedens_nitroreducens_ANME_2d_ GCA_000685155.1|1073875425:0.05010657,MPEBLZ_01201|Candidatus_Methanoperedens_BLZ1_GCA _001317315.1|1103803887:0.08414172)1.000000:0.24033765,((()((MSHOH_0084|Methanosarcina_hor onobensis_HB_1_JCM_15518_GCA_000970285.1|1012765989:0.01207746,(MmTuc01_1281|Methanos arcina_mazei_Tuc01_GCA_000341715.1|1007416496:0.00288654,(MSMAW_0076|Methanosarcina_m azei_WWM610_GCA_000970165.1|1012746205:9.72901197747427e-

07,((MSMAC_0072|Methanosarcina_mazei_C16_GCA_000970245.1|1012759384:0.00183533,(MSMAP _0074|Methanosarcina_mazei_SarPi_GCA_000970185.1|1012749481:6.71122488318732e-

07,MSMAL_0075|Methanosarcina_mazei_LYC_GCA_000970225.1|1012756040:9.94773910438667e07)0.000000:7.86115479000781e-07)0.000000:7.54339397223019e-

07,(((MSLAZ_0156|Methanosarcina_lacustris_Z_7289_GCA_000970265.1|1012762839:0.03605193,(MS MTP_0100|Methanosarcina_MTP4_MTP4_GCA_000970045.1|1012727238:0.06722702,(MSWH1_0089 |Methanosarcina_WH1_WH1_GCA_000970005.1|1012720511:8.49352497523091e07,MSWHS_0092|Methanosarcina_WWM596_WWM596_GCA_000969965.1|1012713361:7.08715830 035093 -

07)0.999873:0.01583572)1.000000:0.04641949)1.000000:0.02162272,(MA_4546|Methanosarcina_acet ivorans_C2A_GCA_000007345.1|1000110087:0.00746893,(MSSAC_4255|Methanosarcina_siciliae_C2J_ GCA_000970145.1|1012745983:7.91769307373167e-

07,(MSSIH_3759|Methanosarcina_siciliae_HI350_GCA_000970125.1|1012741587:6.36869378522375e- 
07,MSSIT_3838|Methanosarcina_siciliae_T4_M_GCA_000970085.1|1012737695:6.21192315817165e07)0.000000:8.44053338422121e-

07)0.987850:0.00360667)0.999999:0.01097102)0.982894:0.00497407,(MM_1240|Methanosarcina_ma zei_Go1_GCA_000007065.1|1000071851:8.08536144402852e-

07,MSMAS_0073|Methanosarcina_mazei_S_6_GCA_000970205.1|1012752691:5.39562769378932e-

07)1.000000:0.00739657)1.000000:0.01484503)0.000000:9.35602750437177e-

07)0.999985:0.00631511)0.999721:0.00724257)1.000000:0.01442305,(MSVAZ_3413|Methanosarcina_ vacuolata_Z_761_GCA_000969905.1|1012707116:0.02977471,MSKOL_3374|Methanosarcina_Kolksee_ Kolksee_GCA_000969945.1|1012713134:0.00750188)0.999999:0.00867310)0.610632:0.00674378,(MS BR3_2340|Methanosarcina_barkeri_3_GCA_000970305.1|1012772340:0.00402966,(MSBRM_0101|Me thanosarcina_barkeri_MS_GCA_000970025.1|1012723697:5.80205604830601e-

07,(MCM1_2594|Methanosarcina_barkeri_CM1_GCA_001027005.1|1013430301:8.47749211436076e07,MSBR2_2279|Methanosarcina_barkeri_227_GCA_000970065.1|1012732645:5.73175608369787e07)0.000000:9.08475737970338e-

07)1.000000:0.01592937)0.895806:0.00753637)0.950341:0.01085955,(Mbar_A0893|Methanosarcina_b arkeri_Fusaro_GCA_000195895.1|1004611845:6.12210952296577e-

07,MSBRW_0105|Methanosarcina_barkeri_Wiesmoor_GCA_000969985.1|1012716761:5.93255738255 $348 \mathrm{e}-$

07)0.870688:0.00498912)1.000000:0.02784699,(MSTHC_1645|Methanosarcina_thermophila_CHTI_55_ GCA_000969925.1|1012708893:0.01247491,MSTHT_1644|Methanosarcina_thermophila_TM_1_GCA_ 000969885.1|1012702824:0.00643124)0.993164:0.01707125)1.000000:0.08928093,(Mzhil_0857|Meth anosalsum_zhilinae_DSM_4017_GCA_000217995.1|1005115425:0.07138347,(Metev_0902|Methanoha lobium_evestigatum_Z_7303_GCA_000196655.1|1004685691:0.15547920,((Mpsy_0828|Methanolobus _psychrophilus_R15_GCA_000306725.1|1006877406:0.04419977,Metho_1670|Methanomethylovoran s_hollandica_DSM_15978_GCA_000328665.1|1007234695:0.05715511)1.000000:0.03106752,(Mmah_ 0612|Methanohalophilus_mahii_DSM_5219_GCA_000025865.1|1003060433:0.12564167,(Mbur_2417 |Methanococcoides_burtonii_DSM_6242_GCA_000013725.1|1001062665:0.02076925,MCMEM_0559| Methanococcoides_methylutens_MM1_GCA_000970325.1|1012774034:0.02872215)0.999965:0.01610 450)0.685701:0.01630581)0.995813:0.02278192)0.459458:0.01227207)1.000000:0.08213469)1.000000 :0.06515452)1.000000:0.06665741)0.999880:0.05561340)1.000000:0.06634783,((RCIX2063|Methanoce Ila_arvoryzae_MRE50_GCA_000063445.1|1003287269:0.04448339,(MCP_0516|Methanocella_paludico la_SANAE_GCA_000011005.1|1000658754:0.02898308,Mtc_0908|Methanocella_conradii_HZ254_GCA _000251105.1|1005860307:0.02614788)0.999998:0.03159327)1.000000:0.11882153,((Mboo_0582|Me thanoregula_boonei_6A8_GCA_000017625.1|1001720368:0.08219294,Metfor_2609|Methanoregula_f ormicica_SMSP_GCA_000327485.1|1007211635:0.07289281)0.793427:0.02295741,(AGIY02000001.1_1 682 |Methanolinea_tarda_NOBI_1_GCA_000235685.3|1905301974:0.08547085,(Mpal_2309|Methanos phaerula_palustris_E1_9c_GCA_000021965.1|1002434548:0.09662543,((Mhun_2148|Methanospirillu m_hungatei_JF_1_GCA_000013445.1|1001012638:0.10749824,(XE11_1766|Methanomicrobiales_arch aeon_53_19_GCA_001508695.1|1115377313:0.05373771,(Mlab_1561|Methanocorpusculum_labreanu m_Z_GCA_000015765.1|1001420341:0.00800580,Mlab_1068|Methanocorpusculum_labreanum_Z_GC A_000015765.1|1001419853:0.01865497)1.000000:0.10766926)1.000000:0.04040046)1.000000:0.0264 5302,((BCNW01000001.1_695|Methanofollis_ethanolicus_HASU_GCA_001571385.1|1904916715:0.02 232893,Metli_0498|Methanofollis_liminatans_DSM_4140_GCA_000275865.1|1027627249:0.02263087 )1.000000:0.02629042,((Mpet_1991|Methanolacinia_petrolearia_DSM_11571_GCA_000147875.1|100 
3776088:0.09679043,(BCNW01000001.1_1265|Methanofollis_ethanolicus_HASU_GCA_001571385.1|1 904917285:0.02406623,Metli_1710|Methanofollis_liminatans_DSM_4140_GCA_000275865.1|1027628 437:0.03233532)0.896044:0.01284846)0.990964:0.01128993,((Memar_0378|Methanoculleus_marisnig ri_JR1_GCA_000015825.1|1001426751:0.02274964,((Memar_0613|Methanoculleus_marisnigri_JR1_G CA_000015825.1|1001426986:0.06540391,(BN140_1738|Methanoculleus_bourgensis_MS2_GCA_0003 04355.2|1006848220:0.00390826,MMAB1_2235|Methanoculleus_MAB1_GCA_900036045.1|1016575 809:0.00359228)0.994326:0.01369394)1.000000:0.05432805,(BN140_1521|Methanoculleus_bourgensi s_MS2_GCA_000304355.2|1006848004:0.00755990,MMAB1_1952|Methanoculleus_MAB1_GCA_9000 36045.1|1016575526:0.00186165)1.000000:0.01939342)0.554139:0.01222869)1.000000:0.03016058,( Metfor_0874|Methanoregula_formicica_SMSP_GCA_000327485.1|1007209964:0.15318232,(Metlim_2 882|Methanoplanus_limicola_DSM_2279_GCA_000243255.1|1024343189:0.03801477,(JOMF0100000 6.1_122|Methanomicrobium_mobile_BP_GCA_000711215.1|1502837525:0.06489704,(Mpet_2415|Me thanolacinia_petrolearia_DSM_11571_GCA_000147875.1|1003776508:0.02373258,BBBG01000001.1_4 08|Methanogenium_cariaci_JCM_10550_GCA_001315945.1|1804947745:0.04651305)1.000000:0.0243 7557)0.999976:0.01336120)0.999999:0.02954641)0.999951:0.02510206)1.000000:0.02312008)1.00000 0:0.03565873)0.889024:0.01517694)1.000000:0.04425541)1.000000:0.04285552)0.999758:0.03469839 )1.000000:0.18324183)0.999445:0.07171764)1.000000:0.13596907,((((ALG76131.1|uncultured_archae on|933572089:0.03245354,(ANME1_1|ANME1|1008840:0.06102715,GZfos19C7_1|uncultured_archae on_GZfos19C7|52548862:0.04246399)1.000000:0.08321825)1.000000:0.51228200,(KYC53403.1|Arc_I group_archaeon_U1lsi0528_Bin055|22222:0.01642542,(ADurb1213_1|Arc_I_group_archaeon_ADurb1 213_Bin02801|1004833076:0.01424631,(B15fssc0709_1|Arc_Igroup_archaeon_B15fssc0709_Meth_Bi n003|1004822508:0.00396075,KYC51830.1|Arc_Igroup_archaeon_U1lsi0528_Bin089|111111:0.00714 622)0.963147:0.00508158)0.639801:0.00703485)1.000000:0.20347278)1.000000:0.11694038,((HMET1 _2442|HMET1|22202442:0.17188908,(AMET1_1458|AMET1|33301458:0.02128561,AMET1_1459|AM ET1|33301459:0.20031344)1.000000:0.12355327)1.000000:0.22075435,((AOA81_01015|Methanomass iliicoccales_archaeon_RumEn_M2_GCA_001421175.1|1105804417:0.07068772,(AUP07_0616|methano genic_archaeon_mixed_culture_ISO4_G1_GCA_001563305.1|1122311422:0.07146645,(Mpt1_c02010| Candidatus_Methanoplasma_termitum_MpT1_GCA_000800805.1|1010972103:0.07722367,(TALC_004 72 |Thermoplasmatales_archaeon_BRNA1_BRNA1_GCA_000350305.1|1007532583:0.03545480,(AR505 _1396|methanogenic_archaeon_ISO4_H5_ISO4_H5_GCA_001560915.1|1016311450:0.10754099,MMA LV_04000|Candidatus_Methanomethylophilus_alvus_Mx1201_GCA_000300255.2|1006808093:0.0222 7299)0.977774:0.01442522)1.000000:0.08387344)0.986484:0.02240727)1.000000:0.04915152)1.00000 0:0.16748184,(MMINT_15640|Candidatus_Methanomassiliicoccus_intestinalis_Issoire_Mx1_GCA_0004 04225.1|1007750285:0.06447152,LFRW01000043.1_38|Methanomicrobia_archaeon_DTU008_GCA_00 1512965.1|1902491087:0.05692449)0.917929:0.03517447)1.000000:0.12578774)0.999999:0.07990573 )0.999992:0.06170473,((V4_1|Archaeon_V4|1060715800:0.05330170,(V3_1|Archaeon_V3|106071570 6:0.03074476,(V2_1|Archaeon_V2|33333:8.17491168740448e-

07,V1_1|Archaeon_V1|44444:7.86425300829521e-

07)1.000000:0.02496784)1.000000:0.06219962)1.000000:0.16349415,(AOA66_1761|Bathyarchaeota_a rchaeon_BA2_GCA_001399795.1|1104202758:0.12113547,AOA65_0408|Bathyarchaeota_archaeon_BA 1_GCA_001399805.1|1104204812:0.23441402)1.000000:1.21197221)0.979842:0.05344801)0.999971:0 $.05362890) 0.559078: 0.03427466) 1.000000: 0.07271174) 1.000000: 0.07011083) 1.000000: 0.05404140,(($ Mvol_1128|Methanococcus_voltae_A3_GCA_000006175.2|1000009600:0.03559769,(Mevan_0872|Me thanococcus_vannielii_SB_GCA_000017165.1|1001660910:0.03848973,(MmarC6_1111|Methanococcu 
S_maripaludis_C6_GCA_000018485.1|1001863981:8.1418684440303e-

07,(MmarC7_0806|Methanococcus_maripaludis_C7_GCA_000017225.1|1001670302:0.00375769,(Mm arC5_0017|Methanococcus_maripaludis_C5_GCA_000016125.1|1001478252:0.00874343,(MMP1559|

Methanococcus_maripaludis_S2_GCA_000011585.1|1000733994:6.07842348502832e-

07,GYY_08645|Methanococcus_maripaludis_X1_GCA_000220645.1|1005244998:0.00378011)1.000000 $: 0.02600065) 0.999999: 0.01144720) 0.962063: 0.00381072) 0.938709: 0.00623125) 1.000000: 0.03439419)$ 1.000000:0.06660298,(Metok_0956|Methanothermococcus_okinawensis_IH1_GCA_000179575.2|1004 206535:0.04647616,Maeo_1268|Methanococcus_aeolicus_Nankai_3_GCA_000017185.1|1001662977: 0.06989724)0.999967:0.03166665)1.000000:0.03604865)0.999784:0.02343538)1.000000:0.03124226,( MJ_0846|Methanocaldococcus_jannaschii_DSM_2661_GCA_000091665.1|1003421250:6.82530054572 $775 \mathrm{e}-$

07,(Mefer_0938|Methanocaldococcus_fervens_AG86_GCA_000023985.1|1002762070:0.01533686,MF S40622_1315|Methanocaldococcus_FS406_22_FS406_22_GCA_000025525.1|1003011202:0.00763231) 0.999063:0.00567780)0.997933:0.00895298)1.000000:0.01807902)0.430311:0.00133811); 


\section{Supplementary Data 3. Phylogenetic tree of McrA subunit (FastTree method) in Newick format.}

The maximum-likelihood phylogenetic tree of the McrA proteins was built using the FastTree program as described in the Materials and Methods section. Sequence set and underlying multiple alignment, which can be provided by a request, are the same as on the Figure 3B.

The tree (below) is provided in Newick format, could be open in MEGA or in any other tree visualization software. To open the file copy the text below the dotted line to a text file with "tre" extension. MEGA (or other tree visualization software) should open the file automatically.

(Metin_0281|Methanocaldococcus_infernus_ME_GCA_000092305.1| 1003473330:0.02703,JH146_1056 | Methanocaldococcus_bathoardescens_JH146_GCA_000739065.1| 1009883047:0.01351,(Metvu_0149|Methanocaldococcus_vulcanius_M7_GCA_000024625.1| 1002866825:0.00195,((Metig_1237|Methanot orris_igneus_Kol_5_GCA_000214415.1| 1005029408:0.02262,((((Metig_0445|Methanotorris_igneus_Ko I_5_GCA_000214415.1| 1005028635:0.03124,(Metvu_1194|Methanocaldococcus_vulcanius_M7_GCA_000024625.1| 1002867860:0.01200,(JH146_0503|Methanocaldococcus_bathoardescens_JH146_GCA_0 00739065.1| 1009882501:0.01149,(MFS40622_0424|Methanocaldococcus_FS406_22_FS406_22_GCA_0 00025525.1|1003010328:0.00544,MJ_0083|Methanocaldococcus_jannaschii_DSM_2661_GCA_000091 665.1|1003420464:0.00229)0.852:0.00568)0.731:0.01123)0.980:0.02612)0.924:0.02541,((Mfer_0734| Methanothermus_fervidus_DSM_2088_GCA_000166095.1|1003993923:0.07107,(MTBMA_c15120|Met hanothermobacter_marburgensis_Marburg_GCA_000145295.1|1003678604:0.00481,(MTCT_1022|Met hanothermobacter_CaT2_CaT2_GCA_000828575.1|1011387870:0.00188,MTH_1129|Methanothermob acter_thermautotrophicus_Delta_H_GCA_000008645.1|1000281338:0.00015)0.860:0.00464)1.000:0.06 629)0.954:0.03050,((Metbo_2281|Methanobacterium_lacus_AL_21_GCA_000191585.1|1004476749:0. 11233,(MBMB1_1825|Methanobacterium_MB1_GCA_000499765.1|1008479935:0.01170,((BRM9_215 6|Methanobacterium_formicicum_BRM9_GCA_000762265.1|1010556959:6.54299095309854e07,MB9_2187|Methanobacterium_formicicum_GCA_001458655.1|1015170296:7.17998872207076e07):0.00014,DSM1535_1095|Methanobacterium_formicicum_GCA_000953115.1|1012186986:0.00186) 0.935:0.01602)0.995:0.05400)0.964:0.04567,(Msp_0321|Methanosphaera_stadtmanae_DSM_3091_GC A_000012545.1|1000868365:0.13292,(YLM1_1111|Methanobrevibacter_olleyae_YLM1_GCA_0015632 45.1|1016364553:0.07780,(Msm_0902|Methanobrevibacter_smithii_ATCC_35061_GCA_000016525.1| 1001555353:0.06012,sm9_1353|Methanobrevibacter_millerae_SM9_GCA_001477655.1|1015276403:0 .05799)0.906:0.03771)0.998:0.07656)0.843:0.03159)0.846:0.03554)0.955:0.04172)0.998:0.06809,(MK0 655|Methanopyrus_kandleri_AV19_GCA_000007185.1|1000090767:0.16447,(((XD44_1486x|Methano bacteriaceae_archaeon_41_258_GCA_001507955.1|1115316079:0.07260,((MSWAN_2056|Methanoba cterium_paludis_SWAN1_GCA_000214725.1|1005042534:0.05628,((Metbo_0353|Methanobacterium_I acus_AL_21_GCA_000191585.1|1004474859:0.05643,((BRM9_0935|Methanobacterium_formicicum_B RM9_GCA_000762265.1|1010555753:7.15783904442167e-

07,MB9_1989|Methanobacterium_formicicum_GCA_001458655.1|1015170110:8.44361486656883e-

07,DSM1535_0905|Methanobacterium_formicicum_GCA_000953115.1|1012186808:7.6858066087019 $4 \mathrm{e}-$

07):0.00864,MBMB1_1682|Methanobacterium_MB1_GCA_000499765.1|1008479804:0.00827)0.999:0. 05491)0.977:0.03751,(Abm4_1526|Methanobrevibacter_AbM4_AbM4_GCA_000404165.1|100774382 
7:0.10389,((mru_1924|Methanobrevibacter_ruminantium_M1_GCA_000024185.1|1002801036:0.0314 3,YLM1_1379|Methanobrevibacter_olleyae_YLM1_GCA_001563245.1|1016364821:0.02213)1.000:0.06 629,(Msm_1015|Methanobrevibacter_smithii_ATCC_35061_GCA_000016525.1|1001555466:0.05416,( TL18_09320|Methanobrevibacter_YE315_YE315_GCA_001548675.1|1016086870:0.02878,sm9_2027| Methanobrevibacter_millerae_SM9_GCA_001477655.1|1015277052:0.01683)0.908:0.01289)0.931:0.02 911)0.991:0.04765)1.000:0.07611)0.369:0.01004)0.988:0.04014,(MTH_1164|Methanothermobacter_th ermautotrophicus_Delta_H_GCA_000008645.1|1000281373:0.00195,(MTCT_1058|Methanothermobac ter_CaT2_CaT2_GCA_000828575.1|1011387906:0.00190,MTBMA_c15480|Methanothermobacter_mar burgensis_Marburg_GCA_000145295.1|1003678640:0.00379)0.743:0.00182)0.987:0.03076)0.997:0.053 06)0.983:0.05530,Mfer_0784|Methanothermus_fervidus_DSM_2088_GCA_000166095.1|1003993973: 0.09697)1.000:0.11536,((((AAQ63481.1|ANME2 |34305116:0.26763,(Mhar_0498|Methanosaeta_harun dinacea_6Ac_GCA_000235565.1|1005532317:0.10446,(Mthe_0569|Methanosaeta_thermophila_PT_G CA_000014945.1|1001273562:0.04212,MCON_0759|Methanosaeta_concilii_GP6_GCA_000204415.1|1 004825313:0.09481)0.432:0.02365)1.000:0.12167)0.998:0.10615,((XD46_0340|Euryarchaeota_archaeo n_55_53_GCA_001507975.1|1115318852:0.00014,(JONQ01000011.1_86|Methermicoccus_shengliensis _DSM_18856_GCA_000711905.1|1502897550:8.06307493934575e-

07,XD62_0880|Methanosarcinales_archeaon_56_1174_GCA_001508185.1|1115337013:6.1737767881 $5655 \mathrm{e}-$

07):0.00014)1.000:0.17281,((MPEBLZ_01201|Candidatus_Methanoperedens_BLZ1_GCA_001317315.1| 1103803887:0.08163,ANME2D_01104|Candidatus_Methanoperedens_nitroreducens_ANME_2d_GCA_ 000685155.1|1073875425:0.04893)1.000:0.23328,((((MSWHS_0092|Methanosarcina_WWM596_WW M596_GCA_000969965.1|1012713361:8.37240504237837e-

07,MSWH1_0089|Methanosarcina_WH1_WH1_GCA_000970005.1|1012720511:7.776862860148e07):0.01733,MSMTP_0100|Methanosarcina_MTP4_MTP4_GCA_000970045.1|1012727238:0.06504)0.9 30:0.03118,((((MSMAC_0072|Methanosarcina_mazei_C16_GCA_000970245.1|1012759384:0.00184,( MSMAL_0075|Methanosarcina_mazei_LYC_GCA_000970225.1|1012756040:9.31772611599058e07,MSMAP_0074|Methanosarcina_mazei_SarPi_GCA_000970185.1|1012749481:9.62409953129187e07,MSMAW_0076|Methanosarcina_mazei_WWM610_GCA_000970165.1|1012746205:9.16928765824 867e-

07):0.00014)0.925:0.00015,(MmTuc01_1281|Methanosarcina_mazei_Tuc01_GCA_000341715.1|10074 16496:0.00367,MSHOH_0084|Methanosarcina_horonobensis_HB_1_JCM_15518_GCA_000970285.1|1 012765989:0.01873)0.709:0.00557)0.932:0.01180,((MSMAS_0073|Methanosarcina_mazei_S_6_GCA_0 00970205.1|1012752691:5.07415726213816e-

07,MM_1240|Methanosarcina_mazei_Go1_GCA_000007065.1|1000071851:9.5615223938956e-

07):0.00746,((MSSIT_3838|Methanosarcina_siciliae_T4_M_GCA_000970085.1|1012737695:7.3213979 $1398593 \mathrm{e}-$

07,MSSIH_3759|Methanosarcina_siciliae_HI350_GCA_000970125.1|1012741587:9.27944943352539e07,MSSAC_4255|Methanosarcina_siciliae_C2J_GCA_000970145.1|1012745983:9.00286247473931e07):0.00362,MA_4546|Methanosarcina_acetivorans_C2A_GCA_000007345.1|1000110087:0.00747)0.9 94:0.01509)0.763:0.00308)0.174:0.00638,((((MSBRW_0105|Methanosarcina_barkeri_Wiesmoor_GCA_ 000969985.1|1012716761:8.51644064124699e-

07,Mbar_A0893|Methanosarcina_barkeri_Fusaro_GCA_000195895.1|1004611845:8.87665177378244e

07):0.01089,(MSBR3_2340|Methanosarcina_barkeri_3_GCA_000970305.1|1012772340:0.00716,(MSBR 
2_2279|Methanosarcina_barkeri_227_GCA_000970065.1|1012732645:5.59179309559571e07,MSBRM_0101|Methanosarcina_barkeri_MS_GCA_000970025.1|1012723697:9.18986902605818e07,MCM1_2594|Methanosarcina_barkeri_CM1_GCA_001027005.1|1013430301:6.87136427406834e07):0.01260)0.801:0.00972)0.097:0.00521,(MSKOL_3374|Methanosarcina_Kolksee_Kolksee_GCA_0009 69945.1|1012713134:0.00895,MSVAZ_3413|Methanosarcina_vacuolata_Z_761_GCA_000969905.1|10 12707116:0.02778)0.944:0.01363)0.728:0.00512,(MSTHT_1644|Methanosarcina_thermophila_TM_1_G CA_000969885.1|1012702824:0.01187,MSTHC_1645|Methanosarcina_thermophila_CHTI_55_GCA_000 969925.1|1012708893:0.00791)0.998:0.03854)0.953:0.01633)0.845:0.01486,MSLAZ_0156|Methanosar cina_lacustris_Z_7289_GCA_000970265.1|1012762839:0.03978)0.590:0.02537)0.999:0.09648,(((Metho _1670|Methanomethylovorans_hollandica_DSM_15978_GCA_000328665.1|1007234695:0.05559,Mps y_0828|Methanolobus_psychrophilus_R15_GCA_000306725.1|1006877406:0.04434)0.975:0.03051,(( MCMEM_0559|Methanococcoides_methylutens_MM1_GCA_000970325.1|1012774034:0.02855,Mbur _2417|Methanococcoides_burtonii_DSM_6242_GCA_000013725.1|1001062665:0.02042)0.919:0.0162 7,Mmah_0612|Methanohalophilus_mahii_DSM_5219_GCA_000025865.1|1003060433:0.12070)0.304: 0.01499)0.642:0.01850,(Mzhil_0857|Methanosalsum_zhilinae_DSM_4017_GCA_000217995.1|1005115 425:0.07151,Metev_0902|Methanohalobium_evestigatum_Z_7303_GCA_000196655.1|1004685691:0. 14114)0.445:0.01777)0.996:0.07456)0.986:0.06331)0.968:0.05905)0.818:0.05190)0.969:0.06073,((RCIX 2063|Methanocella_arvoryzae_MRE50_GCA_000063445.1|1003287269:0.04191,(Mtc_0908|Methanoc ella_conradii_HZ254_GCA_000251105.1|1005860307:0.02535,MCP_0516|Methanocella_paludicola_SA NAE_GCA_000011005.1|1000658754:0.02992)0.961:0.03416)0.998:0.10871,(((AGIY02000001.1_1682| Methanolinea_tarda_NOBI_1_GCA_000235685.3|1905301974:0.08351,(Mpal_2309|Methanosphaerula _palustris_E1_9c_GCA_000021965.1|1002434548:0.09091,((Mhun_2148|Methanospirillum_hungatei_J F_1_GCA_000013445.1|1001012638:0.10439,(XE11_1766|Methanomicrobiales_archaeon_53_19_GCA _001508695.1|1115377313:0.04978,(Mlab_1068|Methanocorpusculum_labreanum_Z_GCA_00001576 5.1|1001419853:0.01884,Mlab_1561|Methanocorpusculum_labreanum_Z_GCA_000015765.1|100142 0341:0.00756)1.000:0.10445)0.989:0.04046)0.944:0.02629,((Metli_0498|Methanofollis_liminatans_DS M_4140_GCA_000275865.1|1027627249:0.02157,BCNW01000001.1_695|Methanofollis_ethanolicus_ HASU_GCA_001571385.1|1904916715:0.02213)0.982:0.02277,((Mpet_1991|Methanolacinia_petroleari a_DSM_11571_GCA_000147875.1|1003776088:0.09208,(Metli_1710|Methanofollis_liminatans_DSM_ 4140_GCA_000275865.1|1027628437:0.03023,BCNW01000001.1_1265|Methanofollis_ethanolicus_HA SU_GCA_001571385.1|1904917285:0.02504)0.556:0.01281)0.779:0.01050,((((Memar_0613|Methanoc ulleus_marisnigri_JR1_GCA_000015825.1|1001426986:0.06466,(MMAB1_2235|Methanoculleus_MAB1 _GCA_900036045.1|1016575809:0.00446,BN140_1738|Methanoculleus_bourgensis_MS2_GCA_00030 4355.2|1006848220:0.00296)0.851:0.01311)1.000:0.05248,(MMAB1_1952|Methanoculleus_MAB1_GC A_900036045.1|1016575526:0.00179,BN140_1521|Methanoculleus_bourgensis_MS2_GCA_00030435 5.2|1006848004:0.00749)0.977:0.01949)0.213:0.01193,Memar_0378|Methanoculleus_marisnigri_JR1_ GCA_000015825.1|1001426751:0.02281)0.988:0.03234,(Metfor_0874|Methanoregula_formicica_SMS P_GCA_000327485.1|1007209964:0.14563,(Metlim_2882|Methanoplanus_limicola_DSM_2279_GCA_0 00243255.1|1024343189:0.03838,(JOMF01000006.1_122|Methanomicrobium_mobile_BP_GCA_00071 1215.1|1502837525:0.06290,(BBBG01000001.1_408|Methanogenium_cariaci_JCM_10550_GCA_00131 5945.1|1804947745:0.04547,Mpet_2415|Methanolacinia_petrolearia_DSM_11571_GCA_000147875.1 |1003776508:0.02372)0.990:0.02468)0.902:0.01269)0.977:0.02937)0.803:0.02180)0.980:0.02213)0.989 :0.03669)0.438:0.01428)0.964:0.04505)0.956:0.04177)0.981:0.04392,Mboo_0582|Methanoregula_boo nei_6A8_GCA_000017625.1|1001720368:0.07759)0.142:0.02079,Metfor_2609|Methanoregula_formici 
ca_SMSP_GCA_000327485.1|1007211635:0.06207)1.000:0.17283)0.846:0.07042)1.000:0.12508,((((ALG 76131.1|uncultured_archaeon|933572089:0.03413,(GZfos19C7_1|uncultured_archaeon_GZfos19C7|5 2548862:0.04207,ANME1_1|ANME1|1008840:0.06005)0.994:0.07908)1.000:0.44995,(KYC53403.1|Arc_ I_group_archaeon_U1lsi0528_Bin055|22222:0.01451,(ADurb1213_1|Arc_I_group_archaeon_ADurb121 3_Bin02801|1004833076:0.01410,(KYC51830.1|Arc_I_group_archaeon_U1lsi0528_Bin089|111111:0.00 704,B15fssc0709_1|Arc_I_group_archaeon_B15fssc0709_Meth_Bin003|1004822508:0.00407)0.763:0.0 0509)0.742:0.00896)1.000:0.18484)0.995:0.10399,((HMET1_2442|HMET1|22202442:0.16218,(AMET1_ 1459|AMET1|33301459:0.18755,AMET1_1458|AMET1|33301458:0.01960)0.997:0.11910)1.000:0.2011 9,((AOA81_01015|Methanomassiliicoccales_archaeon_RumEn_M2_GCA_001421175.1|1105804417:0.0 6302,(AUP07_0616|methanogenic_archaeon_mixed_culture_ISO4_G1_GCA_001563305.1|1122311422 :0.06817,(Mpt1_c02010|Candidatus_Methanoplasma_termitum_MpT1_GCA_000800805.1|101097210 3:0.07352,(TALC_00472|Thermoplasmatales_archaeon_BRNA1_BRNA1_GCA_000350305.1|100753258 3:0.03514,(MMALV_04000|Candidatus_Methanomethylophilus_alvus_Mx1201_GCA_000300255.2|100 6808093:0.02259,AR505_1396|methanogenic_archaeon_ISO4_H5_ISO4_H5_GCA_001560915.1|10163 11450:0.10176)0.708:0.01363)1.000:0.08113)0.743:0.02297)0.977:0.04980)1.000:0.15946,(LFRW01000 043.1_38|Methanomicrobia_archaeon_DTU008_GCA_001512965.1|1902491087:0.05316,MMINT_156 40|Candidatus_Methanomassiliicoccus_intestinalis_Issoire_Mx1_GCA_000404225.1|1007750285:0.064 91)0.551:0.03432)0.979:0.11243)0.969:0.07224)0.917:0.05947,((V4_1|Archaeon_V4|1060715800:0.050 56,((V1_1|Archaeon_V1|44444:9.33967704786324e-

07,V2_1|Archaeon_V2|33333:7.68190866327199e-

07):0.02501,V3_1|Archaeon_V3|1060715706:0.03080)0.998:0.06365)0.999:0.15213,(AOA65_0408|Bat hyarchaeota_archaeon_BA1_GCA_001399805.1|1104204812:0.20816,AOA66_1761|Bathyarchaeota_ar chaeon_BA2_GCA_001399795.1|1104202758:0.11943)1.000:0.95854)0.878:0.05115)0.937:0.04935)0.0 65:0.03582)0.991:0.06674)0.996:0.07024)0.995:0.05553,((Mvol_1128|Methanococcus_voltae_A3_GCA _000006175.2|1000009600:0.03616,((MmarC6_1111|Methanococcus_maripaludis_C6_GCA_00001848 5.1|1001863981:0.00014,(MmarC5_0017|Methanococcus_maripaludis_C5_GCA_000016125.1|100147 8252:0.01885,MmarC7_0806|Methanococcus_maripaludis_C7_GCA_000017225.1|1001670302:0.0037 4)0.732:0.00364)0.808:0.00451,((GYY_08645|Methanococcus_maripaludis_X1_GCA_000220645.1|1005 244998:0.00015,MMP1559|Methanococcus_maripaludis_S2_GCA_000011585.1|1000733994:0.00365) 0.996:0.02441,Mevan_0872|Methanococcus_vannielii_SB_GCA_000017165.1|1001660910:0.03499)0.5 80:0.00734)0.993:0.03427)0.999:0.06296,(Maeo_1268|Methanococcus_aeolicus_Nankai_3_GCA_0000 17185.1|1001662977:0.06452,Metok_0956|Methanothermococcus_okinawensis_IH1_GCA_000179575 $.2 \mid 1004206535: 0.04738) 0.663: 0.03032) 0.987: 0.03649) 0.815: 0.02205) 0.988: 0.03239,(M J+0846 \mid$ Methan ocaldococcus_jannaschii_DSM_2661_GCA_000091665.1|1003421250:0.00015,(MFS40622_1315|Meth anocaldococcus_FS406_22_FS406_22_GCA_000025525.1|1003011202:0.00764,Mefer_0938|Methanoc aldococcus_fervens_AG86_GCA_000023985.1|1002762070:0.01535)0.923:0.00567)0.884:0.01006)0.97 2:0.01699)0.751:0.00156); 


\section{Supplementary Data 4. Phylogenetic tree of McrA subunit (RAxML method) in Newick format.}

The maximum-likelihood phylogenetic tree of the McrA proteins was built using the RAxML program as described in the Materials and Methods section. Sequence set and underlying multiple alignment, which can be provided by a request, are the same as on the Figure 3B.

The tree (below) is provided in Newick format, could be open in MEGA or in any other tree visualization software. To open the file copy the text below the dotted line to a text file with "tre" extension. MEGA (or other tree visualization software) should open the file automatically.

(()((AOA66_1761|Bathyarchaeota_archaeon_BA2_GCA_001399795.1|1104202758:0.120987794624441 00852,AOA65_0408|Bathyarchaeota_archaeon_BA1_GCA_001399805.1|

1104204812:0.234282894192 29031409):1.21096892979541714830,((()((((Mvol_1128|

Methanococcus_voltae_A3_GCA_000006175.2 |1000009600:0.03557107547271076697,(Mevan_0872|

Methanococcus_vannielii_SB_GCA_000017165.1|1001660910:0.03846244468001795702,

(MmarC6_1111|Methanococcus_maripaludis_C6_GCA_0000 18485.1|

1001863981:0.00000091053377919231,(MmarC7_0806|Methanococcus_maripaludis_C7_GCA _000017225.1|1001670302:0.00375499054327142366,(MmarC5_0017|Methanococcus_maripaludis_C 5_GCA_000016125.1|1001478252:0.00873736523554710827,(MMP1559|Methanococcus_maripaludis _S2_GCA_000011585.1|1000733994:0.00000091053377919231,GYY_08645|Methanococcus_maripalu dis_X1_GCA_000220645.1|1005244998:0.00377784727832435789):0.02598155358514374694):0.0114 3934690571796545):0.00380810867820651671):0.00622629122490744348):0.03437080588518088425 ):0.06654952961202062478,(Metok_0956|Methanothermococcus_okinawensis_IH1_GCA_000179575. 2|1004206535:0.04644256905145811160,Maeo_1268|Methanococcus_aeolicus_Nankai_3_GCA_0000 17185.1|1001662977:0.06984731722073268811):0.03164571011885157492):0.0360228044753450951 7,(((MJ_0846|Methanocaldococcus_jannaschii_DSM_2661_GCA_000091665.1|1003421250:0.0000009 1053377919231,(Mefer_0938|Methanocaldococcus_fervens_AG86_GCA_000023985.1|1002762070:0. 01532694144125121977,MFS40622_1315|Methanocaldococcus_FS406_22_FS406_22_GCA_000025525 .1|1003011202:0.00762709603585269569):0.00567408329570758506):0.00894747612475960651,((M etin_0281|Methanocaldococcus_infernus_ME_GCA_000092305.1|1003473330:0.02749645447609468 454,JH146_1056|Methanocaldococcus_bathoardescens_JH146_GCA_000739065.1|1009883047:0.0138 1559875790258803):0.00133772143334417114,Metvu_0149|Methanocaldococcus_vulcanius_M7_GCA _000024625.1|1002866825:0.00191411575348139477):0.01806752824222635292):0.03122144354000 771366,Metig_1237|Methanotorris_igneus_Kol_5_GCA_000214415.1|1005029408:0.02603177792138 764921):0.02342052739217734270):0.05399483645124131259,((Metig_0445|Methanotorris_igneus_K ol_5_GCA_000214415.1|1005028635:0.03085273180765979451,(Metvu_1194|Methanocaldococcus_v ulcanius_M7_GCA_000024625.1|1002867860:0.01454175885490409034,(JH146_0503|Methanocaldoc occus_bathoardescens_JH146_GCA_000739065.1|1009882501:0.01064471376761993125,(MJ_0083|M ethanocaldococcus_jannaschii_DSM_2661_GCA_000091665.1|1003420464:0.00334113174882682933, MFS40622_0424|Methanocaldococcus_FS406_22_FS406_22_GCA_000025525.1|1003010328:0.004407 08763919907959):0.00553224795262899707):0.01095757312879720724):0.02528368106497686646): 0.02342221350344737482,((Mfer_0734|Methanothermus_fervidus_DSM_2088_GCA_000166095.1|10 03993923:0.06979142725357009858,(MTBMA_c15120|Methanothermobacter_marburgensis_Marburg _GCA_000145295.1|1003678604:0.00468736289080738666,(MTH_1129|Methanothermobacter_ther 
mautotrophicus_Delta_H_GCA_000008645.1|1000281338:0.00000091053377919231,MTCT_1022|Met hanothermobacter_CaT2_CaT2_GCA_000828575.1|1011387870:0.00188531647635360559):0.0048232 4368440408859):0.06946022719241358445):0.03225591147718955692,((Metbo_2281|Methanobacter ium_lacus_AL_21_GCA_000191585.1|1004476749:0.11447423372351796245,(MBMB1_1825|Methano bacterium_MB1_GCA_000499765.1|1008479935:0.01103591801033206032,((MB9_2187|Methanobac terium_formicicum_GCA_001458655.1|1015170296:0.00000091053377919231,BRM9_2156|Methano bacterium_formicicum_BRM9_GCA_000762265.1|1010556959:0.00000091053377919231):0.00000091 053377919231,DSM1535_1095|Methanobacterium_formicicum_GCA_000953115.1|1012186986:0.001 86653867632745243):0.01653355209887591090):0.05353127827967996827):0.0481978322737214165 4,(Msp_0321|Methanosphaera_stadtmanae_DSM_3091_GCA_000012545.1|1000868365:0.140506307 83433676330,(YLM1_1111|Methanobrevibacter_olleyae_YLM1_GCA_001563245.1|1016364553:0.079 67831561322247236,(sm9_1353|Methanobrevibacter_millerae_SM9_GCA_001477655.1|1015276403: 0.05783586646839233153,Msm_0902|Methanobrevibacter_smithii_ATCC_35061_GCA_000016525.1|1 001555353:0.06253449471461071385):0.04107483215545691679):0.07689360178260626344):0.03117 711265291037495):0.03510709101174750574):0.04683605721095176339):0.07079381994257956390) $: 0.07007528617133944315$, MK0655|Methanopyrus_kandleri_AV19_GCA_000007185.1|1000090767:0. 18110722712715737748):0.07265866704587634062,(Mfer_0784|Methanothermus_fervidus_DSM_208 8_GCA_000166095.1|1003993973:0.09484401451629621838,(XD44_1486x|Methanobacteriaceae_arc haeon_41_258_GCA_001507955.1|1115316079:0.07451373329720935301,(((Metbo_0353|Methanoba cterium_lacus_AL_21_GCA_000191585.1|1004474859:0.06507090542510356179,(MBMB1_1682|Meth anobacterium_MB1_GCA_000499765.1|1008479804:0.00708854975891557576,((DSM1535_0905|Met hanobacterium_formicicum_GCA_000953115.1|1012186808:0.00000091053377919231,MB9_1989|M ethanobacterium_formicicum_GCA_001458655.1|1015170110:0.00000091053377919231):0.00000091 053377919231,BRM9_0935|Methanobacterium_formicicum_BRM9_GCA_000762265.1|1010555753:0. 00000091053377919231):0.00972096059508046652):0.05201898879523166042):0.0352846517288840 4361,(MSWAN_2056|Methanobacterium_paludis_SWAN1_GCA_000214725.1|1005042534:0.0550102 1655344071754,(Abm4_1526|Methanobrevibacter_AbM4_AbM4_GCA_000404165.1|1007743827:0.10 758876733663678971,((YLM1_1379|Methanobrevibacter_olleyae_YLM1_GCA_001563245.1|10163648 21:0.02052953851792382878,mru_1924|Methanobrevibacter_ruminantium_M1_GCA_000024185.1|1 002801036:0.03334511412900664773):0.06882163791524170682,(Msm_1015|Methanobrevibacter_s mithii_ATCC_35061_GCA_000016525.1|1001555466:0.05356731023882695120,(sm9_2027|Methanob revibacter_millerae_SM9_GCA_001477655.1|1015277052:0.01705198164622686099,TL18_09320|Met hanobrevibacter_YE315_YE315_GCA_001548675.1|1016086870:0.02942884892990814752):0.0142398 0648985330270):0.03031360225946004919):0.04868624359910909971):0.08417498281470943422):0. 01649998311628754488):0.03668466993935488168,(MTH_1164|Methanothermobacter_thermautotr ophicus_Delta_H_GCA_000008645.1|1000281373:0.00191717357578448226,(MTBMA_c15480|Metha nothermobacter_marburgensis_Marburg_GCA_000145295.1|1003678640:0.00378474375040150929, MTCT_1058|Methanothermobacter_CaT2_CaT2_GCA_000828575.1|1011387906:0.0018880809112932 5970):0.00185645097507582276):0.02945810740815567666):0.05688134236942102295):0.059311877 25606764521):0.12235056452273010419):0.03425832396981117606,(((AAQ63481.1|ANME2 |3430511 6:0.28975518354770557483,(Mhar_0498|Methanosaeta_harundinacea_6Ac_GCA_000235565.1|10055 32317:0.10703230448823029819,(MCON_0759|Methanosaeta_concilii_GP6_GCA_000204415.1|10048 25313:0.09652510294879181052,Mthe_0569|Methanosaeta_thermophila_PT_GCA_000014945.1|100 1273562:0.04183939895685286070):0.02374290073383054922):0.12984248393144384726):0.1056655 
6214450607831,((XD46_0340|Euryarchaeota_archaeon_55_53_GCA_001507975.1|1115318852:0.0000 0091053377919231,(XD62_0880|Methanosarcinales_archeaon_56_1174_GCA_001508185.1|11153370 13:0.00000091053377919231,JONQ01000011.1_86|Methermicoccus_shengliensis_DSM_18856_GCA_0 00711905.1|1502897550:0.00000091053377919231):0.00000091053377919231):0.1848208291881800 4691,((ANME2D_01104|Candidatus_Methanoperedens_nitroreducens_ANME_2d_GCA_000685155.1|1 073875425:0.04998351970135428424,MPEBLZ_01201|Candidatus_Methanoperedens_BLZ1_GCA_001 317315.1|1103803887:0.08416528873105851016):0.23955559599015843752,((((MSVAZ_3413|Metha nosarcina_vacuolata_Z_761_GCA_000969905.1|1012707116:0.02820253180315138858,MSKOL_3374| Methanosarcina_Kolksee_Kolksee_GCA_000969945.1|1012713134:0.00900230165605795871):0.00819 297582217770430,((MSBR3_2340|Methanosarcina_barkeri_3_GCA_000970305.1|1012772340:0.00353 248501554328295,(MSBRM_0101|Methanosarcina_barkeri_MS_GCA_000970025.1|1012723697:0.000 00091053377919231,(MCM1_2594|Methanosarcina_barkeri_CM1_GCA_001027005.1|1013430301:0.0 0000091053377919231,MSBR2_2279|Methanosarcina_barkeri_227_GCA_000970065.1|1012732645:0. 00000091053377919231):0.00000091053377919231):0.01627783594733967984):0.0097633508945253 9870,(MSHOH_0084|Methanosarcina_horonobensis_HB_1_JCM_15518_GCA_000970285.1|101276598 9:0.01135890548385280231,(MmTuc01_1281|Methanosarcina_mazei_Tuc01_GCA_000341715.1|1007 416496:0.00361128735510421982,((MSMAC_0072|Methanosarcina_mazei_C16_GCA_000970245.1|10 12759384:0.00183418264182172051,(MSMAW_0076|Methanosarcina_mazei_WWM610_GCA_000970 165.1|1012746205:0.00000091053377919231,(MSMAP_0074|Methanosarcina_mazei_SarPi_GCA_000 970185.1|1012749481:0.00000091053377919231,MSMAL_0075|Methanosarcina_mazei_LYC_GCA_00 0970225.1|1012756040:0.00000091053377919231):0.00000091053377919231):0.00000091053377919 231):0.00000091053377919231,(((MSLAZ_0156|Methanosarcina_lacustris_Z_7289_GCA_000970265.1| 1012762839:0.03602067433402576790,(MSMTP_0100|Methanosarcina_MTP4_MTP4_GCA_00097004 5.1|1012727238:0.06718058595080947382,(MSWH1_0089|Methanosarcina_WH1_WH1_GCA_000970 005.1|1012720511:0.00000091053377919231,MSWHS_0092|Methanosarcina_WWM596_WWM596_ GCA_000969965.1|1012713361:0.00000091053377919231):0.01582584351931447253):0.0463968141 9847564714):0.02160578543522531647,(MA_4546|Methanosarcina_acetivorans_C2A_GCA_00000734 5.1|1000110087:0.00746353787754683827,(MSSIH_3759|Methanosarcina_siciliae_HI350_GCA_00097 0125.1|1012741587:0.00000091053377919231,(MSSIT_3838|Methanosarcina_siciliae_T4_M_GCA_000 970085.1|1012737695:0.00000091053377919231,MSSAC_4255|Methanosarcina_siciliae_C2J_GCA_00 0970145.1|1012745983:0.00000091053377919231):0.00000091053377919231):0.00360526654630763 431):0.01096445464735513499):0.00497006647907133510,(MM_1240|Methanosarcina_mazei_Go1_G CA_000007065.1|1000071851:0.00000091053377919231,MSMAS_0073|Methanosarcina_mazei_S_6_ GCA_000970205.1|1012752691:0.00000091053377919231):0.00739132097357282202):0.0148340479 6877860546):0.00558983002660480566):0.00731407409472270564):0.01367825530080566017):0.003 54159064710375324):0.00965406835990705731,(Mbar_A0893|Methanosarcina_barkeri_Fusaro_GCA_ 000195895.1|1004611845:0.00000091053377919231,MSBRW_0105|Methanosarcina_barkeri_Wiesmo or_GCA_000969985.1|1012716761:0.00000091053377919231):0.01089981403267736328):0.02536162 965728185753,(MSTHC_1645|Methanosarcina_thermophila_CHTI_55_GCA_000969925.1|1012708893: $0.01453976770988349952, M S T H T$ 1644 | Methanosarcina_thermophila_TM_1_GCA_000969885.1|101 2702824:0.00431017328196665883):0.01581991198704868484):0.08864416040078154646,(Mzhil_085 7|Methanosalsum_zhilinae_DSM_4017_GCA_000217995.1|1005115425:0.06821975260970133959,(M etev_0902|Methanohalobium_evestigatum_Z_7303_GCA_000196655.1|1004685691:0.155244176295 82024092,((Mpsy_0828|Methanolobus_psychrophilus_R15_GCA_000306725.1|1006877406:0.0438352 
2512181124708,Metho_1670|Methanomethylovorans_hollandica_DSM_15978_GCA_000328665.1|10 07234695:0.05748456249655778394):0.03091268415469362180,(Mmah_0612|Methanohalophilus_m ahii_DSM_5219_GCA_000025865.1|1003060433:0.12598105265345729831,(Mbur_2417|Methanococc oides_burtonii_DSM_6242_GCA_000013725.1|1001062665:0.02116462055968121314,MCMEM_0559| Methanococcoides_methylutens_MM1_GCA_000970325.1|1012774034:0.02829594195150634053):0. 01604076486992170314):0.01650735861239281330):0.02317182402739481964):0.0147206746854505 5872):0.08340914800102557092):0.06220177512702656369):0.06787191302744061128):0.055566491 83685145182):0.06628718888952939148,((RCIX2063|Methanocella_arvoryzae_MRE50_GCA_00006344 5.1|1003287269:0.04443654020208038374,(MCP_0516|Methanocella_paludicola_SANAE_GCA_00001 1005.1|1000658754:0.02896338555910545412,Mtc_0908|Methanocella_conradii_HZ254_GCA_00025 1105.1|1005860307:0.02613059474945970267):0.03159017677276959590):0.11875807277855891797 ,((Mboo_0582|Methanoregula_boonei_6A8_GCA_000017625.1|1001720368:0.0821323725616696365 1,Metfor_2609|Methanoregula_formicica_SMSP_GCA_000327485.1|1007211635:0.072851245273641 36848):0.02296738353376359329,(AGIY02000001.1_1682|Methanolinea_tarda_NOBI_1_GCA_000235 685.3|1905301974:0.08540821146514238071,(Mpal_2309|Methanosphaerula_palustris_E1_9c_GCA_0 00021965.1|1002434548:0.09655614647583618537,((Mhun_2148|Methanospirillum_hungatei_JF_1_G CA_000013445.1|1001012638:0.10742490016820993581,(XE11_1766|Methanomicrobiales_archaeon_ 53_19_GCA_001508695.1|1115377313:0.05370045222024380505,(Mlab_1561|Methanocorpusculum labreanum_Z_GCA_000015765.1|1001420341:0.00800172069505763160,Mlab_1068|Methanocorpusc ulum_labreanum_Z_GCA_000015765.1|1001419853:0.01864255145029240085):0.1075992281959457 7859):0.04037829603779719695):0.02643091841865416805,((BCNW01000001.1_695|Methanofollis_e thanolicus_HASU_GCA_001571385.1|1904916715:0.02231333905950412533,Metli_0498|Methanofolli s_liminatans_DSM_4140_GCA_000275865.1|1027627249:0.02261680128600441347):0.026272488382 90276167,((Mpet_1991|Methanolacinia_petrolearia_DSM_11571_GCA_000147875.1|1003776088:0.09 672608634084363388,(BCNW01000001.1_1265|Methanofollis_ethanolicus_HASU_GCA_001571385.1| 1904917285:0.02404911488412897133,Metli_1710|Methanofollis_liminatans_DSM_4140_GCA_00027 5865.1|1027628437:0.03231561542065024273):0.01284065903057363470):0.01128162430885408692 ,((Memar_0378|Methanoculleus_marisnigri_JR1_GCA_000015825.1|1001426751:0.0227342351910543 7218,((Memar_0613|Methanoculleus_marisnigri_JR1_GCA_000015825.1|1001426986:0.065360165330 91613956,(BN140_1738|Methanoculleus_bourgensis_MS2_GCA_000304355.2|1006848220:0.0039054 0317578897756,MMAB1_2235|Methanoculleus_MAB1_GCA_900036045.1|1016575809:0.0035904833 4040009114):0.01368673761830612769):0.05429105064520683976,(BN140_1521|Methanoculleus_bo urgensis_MS2_GCA_000304355.2|1006848004:0.00755493191969314106,MMAB1_1952|Methanocull eus_MAB1_GCA_900036045.1|1016575526:0.00186056711245693876):0.01938121330856933805):0.0 1222148292378687218):0.03013927572615051134,(Metfor_0874|Methanoregula_formicica_SMSP_GC A_000327485.1|1007209964:0.15307514535736463368,(Metlim_2882|Methanoplanus_limicola_DSM _2279_GCA_000243255.1|1024343189:0.03799207294170821314,(JOMF01000006.1_122|Methanomi crobium_mobile_BP_GCA_000711215.1|1502837525:0.06485434655959645966,(Mpet_2415|Methano lacinia_petrolearia_DSM_11571_GCA_000147875.1|1003776508:0.02371708628518217829,BBBG0100 0001.1_408|Methanogenium_cariaci_JCM_10550_GCA_001315945.1|1804947745:0.04648664655773 573212):0.02435981324662024952):0.01335046029022032843):0.02952492667168511373):0.0250846 3487809246134):0.02310667356216947535):0.03563784276889413877):0.01516963882851021968):0. 04422477090997305182):0.04283542958073702916):0.03465058511757984627):0.1831289611664433 6586):0.07143754964662794860):0.13610177381130128538):0.05355825861582258485,(((ALG76131.1 
| uncultured_archaeon |933572089:0.03243039693028729187,(ANME1_1|ANME1|1008840:0.0609861 6145761293073,GZfos19C7_1|uncultured_archaeon_GZfos19C7|52548862:0.04244105605716859408) :0.08316382918611400776):0.51186801361038880565,(KYC53403.1|Arc_Igroup_archaeon_U1lsi0528 _Bin055|22222:0.01640990013393240840,(ADurb1213_1|Arc_I_group_archaeon_ADurb1213_Bin0280 1|1004833076:0.01423684310778401858,(B15fssc0709_1|Arc_I_group_archaeon_B15fssc0709_Meth_ Bin003|1004822508:0.00395808066654974519,KYC51830.1|Arc_I_group_archaeon_U1lsi0528_Bin089 |111111:0.00714195465584252596):0.00507818622421484563):0.00703365304065092702):0.2033315 3444948506849):0.11684610588092647632,((HMET1_2442|HMET1|22202442:0.171750871045947411 94,(AMET1_1458|AMET1|33301458:0.02126585186587077320,AMET1_1459|AMET1|33301459:0.200 18119897471053914):0.12348866712463246742):0.22061679207053488949,((AOA81_01015|Methano massiliicoccales_archaeon_RumEn_M2_GCA_001421175.1|1105804417:0.07063622870890796934,(AU P07_0616|methanogenic_archaeon_mixed_culture_ISO4_G1_GCA_001563305.1|1122311422:0.07141 270020160558896,(Mpt1_c02010|Candidatus_Methanoplasma_termitum_MpT1_GCA_000800805.1|1 010972103:0.07716932349529920243,(TALC_00472|Thermoplasmatales_archaeon_BRNA1_BRNA1_GC A_000350305.1|1007532583:0.03542925604718168142,(AR505_1396/methanogenic_archaeon_ISO4_ H5_ISO4_H5_GCA_001560915.1|1016311450:0.10747059805970116120,MMALV_04000|Candidatus_ Methanomethylophilus_alvus_Mx1201_GCA_000300255.2(1006808093:0.02225747707202638606):0.0 1441593641237068346):0.08381627615540536425):0.02239290397469088922):0.04911711838389037 216):0.16735031959178778282,(MMINT_15640|Candidatus_Methanomassiliicoccus_intestinalis_Issoir e_Mx1_GCA_000404225.1|1007750285:0.06442393077017055658,LFRW01000043.1_38|Methanomicr obia_archaeon_DTU008_GCA_001512965.1|1902491087:0.05688486480487258551):0.0351554526018 2888210):0.12567201147009615658):0.07986615670551394652):0.06162823675539848278):0.053407 27315434671413):0.16338590228978372432,V4_1|Archaeon_V4|1060715800:0.053254973557611558 21):0.06216418181374060853,V3_1|Archaeon_V3|1060715706:0.03072433363226454578):0.0249501 0950327556515,V2_1|Archaeon_V2|33333:0.00000091053377919231,V1_1|Archaeon_V1|44444:0.00 000091053377919231); 